\title{
Membrane phosphoinositides stabilize GPCR-arrestin complexes and offer temporal control of complex assembly and dynamics
}

John Janetzko', Ryoji Kise ${ }^{2}$, Benjamin Barsi-Ryne ${ }^{3,4}$, Dirk H. Siepe ${ }^{1,5,6}$, Franziska M. Heydenreich $^{1}$, Matthieu Masureel ${ }^{1,7}$, Kouki Kawakami ${ }^{2}$, K. Christopher Garcia ${ }^{1,5,6}$, Mark von Zastrow $^{3,4}$, Asuka Inoue ${ }^{2^{*}}$, Brian K. Kobilka ${ }^{1,8^{*}}$

Affiliations:

${ }^{1}$ Department of Molecular and Cellular Physiology, Stanford University School of Medicine, Stanford, CA, USA.

${ }^{2}$ Graduate School of Pharmaceutical Sciences, Tohoku University, 6-3, Aoba, Aramaki, Aoba-ku, Sendai, Miyagi, 980-8578 Japan.

${ }^{3}$ Department of Cellular and Molecular Pharmacology, University of California, San Francisco, School of Medicine, San Francisco, CA, USA.

${ }^{4}$ Department of Psychiatry, University of California, San Francisco, School of Medicine, San Francisco, CA, USA.

${ }^{5}$ Department of Structural Biology, Stanford University School of Medicine, Stanford, CA, USA

${ }^{6}$ Howard Hughes Medical Institute, Stanford University School of Medicine, Stanford, CA, USA.

${ }^{7}$ Present address: Department of Structural Biology, Genentech Inc., South San Francisco, CA 94080, USA

${ }^{8}$ Lead contact

*Correspondence: iaska@tohoku.ac.jp (A.I.), kobilka@stanford.edu (B.K.K)

\section{Summary:}

Arrestins recognize activated and phosphorylated G protein-coupled receptors (GPCRs) and are responsible for promoting acute desensitization of receptors as well as their endocytosis. As phosphatidylinositols have been shown to bind to components of the endocytic machinery, including arrestins, we examined the role of phosphoinositide (PIP) binding in GPCR-arrestin complexes. Using a PIP-binding-deficient mutant of arrestin we find that GPCRs stratify into two groups based on whether arrestin-PIP-interactions are required for arrestin recruitment to activated receptors. This requirement for arrestin-PIP-interactions depends on receptor phosphorylation, with receptors having more limited phosphorylation requiring arrestin-PIPbinding capacity. In vitro, this arrestin lipid binding functions to stabilize receptor-arrestin complexes and is crucial for promoting a core-engaged state of the complex. In the absence of a bound GPCR, PIP2, but not endosome resident PI(3)P, promotes conformational changes in arrestin that parallel activation, including movement of the finger and gate loops, but without release of the arrestin $\mathrm{C}$-terminus. These results suggest a model for arrestin recruitment that depends on three components that each function to potentiate the conformation of arrestin: the GPCR core, phosphorylated GPCR C-terminus and membrane phosphoinositides. Integration of a phosphoinositide-dependence into arrestin-GPCR complex assembly provides a mechanism for release of arrestin from GPCRs with insufficient phosphorylation, allowing for their rapid recycling, while explaining how GPCRs that form stable complexes with arrestin can remain associated yet switch from desensitized to allowing $G$ protein coupling in endosomes.

\section{Introduction:}

In order to achieve robust signaling, G protein-coupled receptors (GPCRs) are tightly regulated not just in their activation, but also in their deactivation. GPCR deactivation is a complex multistep process often divided into an acute and a prolonged phase (Rajagopal and Shenoy, 2018).

51 In addition to promoting $G$ protein engagement, agonist stimulation leads to the recruitment of 
GPCR kinases (GRKs), which phosphorylate the receptor and trigger recruitment of arrestins (Komolov and Benovic, 2018). Arrestin serves to first block further $G$ protein engagement, resulting in an acute phase of desensitization, but also to mediate the trafficking of activated receptors to clathrin-coated structures (CCSs) and their internalization. Once internalized, receptors can experience markedly different fates, with some being rapidly recycled to the plasma membrane, while others are retained in intracellular compartments, or directed to lysosomes and degraded (Hanyaloglu and von Zastrow, 2008). In recent years, the discovery that GPCRs can signal from intracellular compartments (Irannejad et al., 2013) has led to a re-framing of GPCR signaling to include not only temporal regulation, but also differences that result from spatially distinct receptor populations (Irannejad et al., 2015; Lobingier and von Zastrow, 2019).

There are four human arrestins; arrestins 1 and 4 are dedicated to the visual system, and arrestins 2 and 3 , also known as $\beta$-arrestin 1 ( $\beta$ arr1) and $\beta$-arrestin 2 ( $\beta$ arr2), respectively are ubiquitously expressed throughout the other tissues of the body. Remarkably, these two $\beta$-arrestins are responsible for recognition and desensitization of hundreds of GPCRs. Though most GPCRs recruit arrestin, the nature and duration of this interaction can differ between receptors, and historically GPCRs have been classified as either a "class A" receptor, which interacts transiently with arrestin, or a "class B" receptor which interacts more stably with arrestin, leading to in cotrafficking of arrestin to endosomes (Oakley et al., 2001; Oakley et al., 2000). Moreover, whether a GPCR interacted transiently or stably with arrestin appeared to correlate with rates of resensitization, with class $A$ receptors re-sensitizing more rapidly than class $B$ receptors (Oakley et al., 1999). Mechanistically, stable association of arrestin to "class B" GPCRs is correlated with the presence of particular phosphorylation site clusters (Oakley et al., 2001); however, it has remained unclear what event precipitates the dissociation of $\beta$-arrestins from "class A" receptors to allow their dephosphorylation and recycling.

Early structural studies into GPCR-arrestin complexes suggested that arrestin could bind to a GPCR either through only the phosphorylated C-terminus (called tail-engaged), or through both the phosphorylated C-terminus and the transmembrane core of the GPCR (called core-engaged) (Shukla et al., 2014). Though unclear what might determine the equilibrium between these states, a tail-engaged state would maintain an accessible GPCR core, possibly allowing it to engage G proteins while bound to arrestin. These so-called "megaplex" assemblies (Nguyen et al., 2019; Thomsen et al., 2016) offered an explanation for sustained CAMP signaling produced by endosomal populations of V2R and PTH1R (Feinstein et al., 2013; Ferrandon et al., 2009), both of which stably associate with $\beta$-arrestins.

At a molecular level, the prevailing model for arrestin activation (and thus recruitment to an active and phosphorylated GPCR) involves displacement of the auto-inhibitory C-terminus of arrestin by the GPCR phosphorylated C-terminus (or in some cases an intracellular loop). Once the arrestin C-terminus has been displaced, a number of structural rearrangements allow for arrestin to engage the GPCR (Sente et al., 2018), including insertion of the arrestin finger loop into a cavity formed by the cytoplasmic ends of transmembrane segments. Recently this model, which suggests a 1:1 interaction, has been challenged by the finding that some "class A" receptors recruit super-stoichiometric quantities of arrestin to the plasma membrane, and can lead to arrestin clustering in CCSs without an associated GPCR (Eichel et al., 2018). This study suggested that arrestin, after dissociation from a GPCR, can maintain an association with the plasma membrane by binding to PIP2 (Eichel et al., 2018), though based on the established mechanism for arrestin activation it was unclear how this would be possible, or how arrestin could promote MAPK signaling from CCSs after GPCR dissociation (Eichel et al., 2016).

100 
102

103

104

105

106

107

108

109

110

111

112

113

114

115

116

117

118

119

120

121

122

123

124

125

126

127

128

129

130

131

132

133

134

135

136

137

138

139

140

141

142

143

144

145

146

147

148

149

150

151
Components of the endocytic machinery such as AP2 (Kadlecova et al., 2017), and $\beta$-arrestins (Gaidarov et al., 1999) have been shown to bind to phosphoinositides. These signaling lipids serve critical functions defining the identity of lipid compartments and acting as coincidence markers for protein-protein recognition and trafficking to occur only in the appropriate subcellular context (De Matteis and Godi, 2004; Di Paolo and De Camilli, 2006). While several studies have investigated the interactions of soluble inositol phosphates with both visual and non-visual arrestins (Chen et al., 2017; Chen et al., 2021; Milano et al., 2006; Zhuang et al., 2010), only one has explored the role of membrane phosphoinositides (Gaidarov et al., 1999). Importantly, this work suggested that plasma membrane PIPs, such as PIP2 and PIP3, may function to stabilize GPCR- $\beta$-arrestin complexes as they traffic to CCSs.

Recent structural studies showing PIP2 bound at the interface between the neurotensin type I receptor (NTSR1) and $\beta$ arr1 (Huang et al., 2020) prompted us to ask the question: "what role do PIPs serve in mediating GPCR- $\beta$-arrestin complex assembly?" Here we show that GPCRs which only transiently engage $\beta$-arrestin require phosphoinositide binding for $\beta$-arrestin recruitment. Further, by using NTSR1 as a model system we find that specific phosphorylation sites are linked to this phosphoinositide binding-dependence for arrestin recruitment. Using in vitro biochemical and biophysical assays, we demonstrate that phosphoinositide binding contributes to the stability of a GPCR- $\beta$-arrestin complex, and in particular promotes a core-engaged state. We also find that phosphoinositides alone are able to promote a partially activated state of arrestin, thereby offering an explanation for how arrestin is able to persist at the plasma membrane once dissociated from a GPCR. Together, these results offer an explanation for how receptors that transiently associate with $\beta$-arrestin are able to recruit (and dissociate) $\beta$-arrestin in a spatiotemporally resolved manner, and strongly coupled receptors are able to maintain a stable association with arrestin in subcellular compartments yet regain the ability for further $G$ protein engagement from subcellular structures.

\section{Results and Discussion:}

\section{Arrestin PIP-binding is important for desensitization of endogenous $\beta 2 A R$}

The PIP-binding-deficient mutant of $\beta$ arr2 (K233Q/R237Q/K251Q, ßarr2 numbering, henceforth $3 Q$, also used to denote mutation of homologous residues in $\beta$ arr1) was previously found to be impaired for internalization of $\beta 2 A R$ (Gaidarov et al., 1999), with $\beta$ arr2 (3Q) failing to traffic to CCSs, though still being recruited from the cytoplasm to the plasma membrane, albeit to a lesser extent than wild-type (WT) (Eichel et al., 2018). As such, we wondered how this behavior effects $\beta 2 A R$ signaling and specifically whether $\beta$ arr2 (3Q) is capable of desensitizing $\beta 2 A R$ at the plasma membrane. Using a recently developed cAMP sensor (Tewson et al., 2016), we monitored cAMP levels in live HEK293 cells lacking both $\beta$-arrestins, and endogenously expressing the $\beta 2 A R$ (O'Hayre et al., 2017). In the absence of exogenously expressed $\beta$ arr2 (transfection of mApple alone), isoproterenol (iso) stimulation led to a sustained cAMP response, while expression of ßarr2-mApple led to expected desensitization. However, expression of ßarr2 (3Q)-mApple resulted in much less desensitization over 30 minutes (Figure S1A); furthermore, this difference was observed in two independent cell lines (Luttrell et al., 2018; O'Hayre et al., 2017). This suggests that the PIP-binding function of $\beta$-arrestins plays an important functional role, in not only internalization (Gaidarov et al., 1999), but also receptor desensitization, and does so under conditions of endogenous GPCR expression.

GPCRs stratify into two groups in their dependence on PIP-binding for arrestin recruitment 
152 That $\beta$ arr2 (3Q) is impaired for recruitment to $\beta 2 A R$, but seemingly not for the chimeric receptor $153 \beta 2 A R-V 2 C$, which bears the C-terminus of the vasopressin V2 receptor (Eichel et al., 2018), 154 suggested that GPCRs may have different dependencies on $\beta$-arrestin PIP-binding capability for 155 recruitment. To investigate this more generally, we used a cell-based NanoBiT assay (Dixon et al., 2016), wherein a plasma membrane marker (CAAX) is fused to the large subunit of a modified

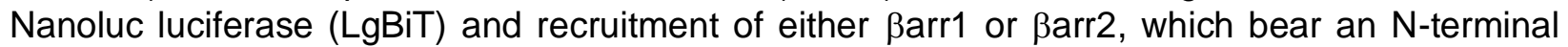
complementary small subunit of Nanoluc ( $\mathrm{SmBiT}$ ), can be monitored by luminescence changes (Figure 1A). We selected a set of 22 representative GPCRs (Supplementary Data Table 1), coexpressed the sensors with each receptor of interest in HEK293 cells, and compared the recruitment of WT $\beta$-arrestin to that of the corresponding $3 Q \beta$-arrestin mutant upon agonist stimulation (Figure 1B, top, Supplementary Data Table 1). We determined time-averaged endpoint luminescence fold-changes from 10-15 minutes post-agonist stimulation and fit the resulting data to generate concentration response curves and extract a recruitment amplitude for each receptor-arrestin pair (see methods) (Figure 1B, bottom, Supplementary Data Figure 1A-B). We then compared the recruitment of WT and $3 \mathrm{Q}$ arrestin using a metric that represented the relative sensitivity of the receptor to loss of arrestin-PIP binding capacity, we termed the loss of function (LOF) index (see methods). Receptors with a low LOF value recruit WT and $3 Q \beta$-arrestins to the plasma membrane similarly, and are deemed PIP-independent, while receptors with a high LOF value show greatly diminished recruitment of $3 Q \quad \beta$-arrestin and are deemed PIP-dependent (Figure 1C). Both WT and $3 Q$ forms of $\beta$ arr1 and $\beta$ arr2 express similarly (Figure S1B, Supplementary Data Figure 2).

173

174

175

176

177

178

179

180

181

182

183

184

185

186

187

188

189

190

191

192

193

194

195

196

197

198

199

200

201
To better understand this distinction, we performed $\mathrm{k}$ means clustering of plasma membrane recruitment data for all receptor- $\beta$-arrestin pairs (Supplementary Data Figures 1 and $6, n=55$ receptor- $\beta$-arrestin pairs), which suggested that the data is best divided into two clusters (see methods, clusters marked by dotted ellipses in Figure 1C). We found only a weak correlation (Pearson correlation $=-0.51 ;-0.4$ when TACR1 and B2R are excluded) between the amplitude of WT arrestin recruitment and the degree of LOF observed (Figure S1C), suggesting that differences in LOF were not simply due to lower levels of WT recruitment. Cluster 1 was defined by receptors that exhibited a high degree of LOF (center LOF $=0.73$ ) and included GPCRs previously classified as "class A" (Oakley et al., 2000): $\beta 2 A R, \mu O R, E T A R, D 1 R, \alpha 1 B R$. Cluster 2 , defined by receptors with a low degree of LOF (center LOF $=0.06$ ), included GPCRs classified as "class B" (Oakley et al., 2000): AT1R, NTSR1, V2R, TRHR, and TACR1. Based on the results from Eichel et al. (Eichel et al., 2018), we tested two chimeric receptors, $\beta 2 A R-V 2 C$ and $\mu O R-$ $\mathrm{V} 2 \mathrm{C}$, both of which showed reduced reliance on $\beta$-arrestin PIP binding capability for plasma membrane recruitment compared to the respective parent receptor. The V1AR, which was previously shown to undergo labile phosphorylation and rapid recycling (Innamorati et al., 1998a; Innamorati et al., 1998b) clusters with the class A receptors in cluster 1, while the V1BR, bearing a closer similarity in its proximal C-terminus to V2R clusters with class B receptors in cluster 2, even though it has been found to only associate transiently with arrestin (Perkovska et al., 2018). In addition, $\beta 1 A R, S 1 P R 1$, and $\delta O R$, all three of which have been suggested to either recycle rapidly or interact transiently with arrestin (Martinez-Morales et al., 2018; Nakagawa and Asahi, 2013; Trapaidze et al., 2000), were assigned to cluster 1. Other receptors known to co-traffic with arrestin to endosomes, including PAR2 (DeFea et al., 2000; Dery et al., 1999; Oakley et al., 2001), B2R (Khoury et al., 2014), and PTH1R (Feinstein et al., 2011) were also classified into cluster 2. Two receptors, OXTR and HTR2C displayed unexpected behavior where $\beta$ arr1 recruitment was dramatically more sensitive to loss of PIP-binding than $\beta$ arr2, resulting in these GPCR- $\beta$-arrestin pairs being divided between the two clusters. OXTR was previously classified as a "class B" receptor (Oakley et al., 2001); however, these studies only examined varr2 recruitment. In the case of the HTR2C, it was reported that PIP2-depletion did not affect association of ßarr2 (Toth 
et al., 2012), which can be consistent with our findings since even though HTR2C was clustered with PIP-dependent receptors the observed LOF of 0.4 for $\beta$ arr2 sits between the two centers. Together, these data show that recruitment of $\beta$-arrestins is dependent on the PIP-binding capacity of arrestin for some GPCRs, but not others, and that this distinction is consistent with the previous class $\mathrm{A} / \mathrm{B}$ categorization.

While our use of a plasma membrane bystander avoids modifying the receptor of interest, we wanted to confirm that plasma membrane recruitment is indeed a reliable proxy for arrestin recruitment to a GPCR of interest, especially given that some receptors such as $\beta 2 A R, \beta 1 A R$, and $\mu \mathrm{OR}$ have been reported to recruit super-stoichiometric quantities of arrestin, relative to receptor (Eichel et al., 2018). For this, we used a direct NanoBiT assay in which the SmBiT component is fused to the $\mathrm{C}$-terminus of each GPCR of interest, and the N-terminus of arrestin is modified with the LgBiT fragment (Figure S1D, left). We found that recruitment measured by this direct complementation largely paralleled recruitment measured using the plasma membrane bystander, with minor exceptions (Supplementary Data Figure 4). Further, directly comparing LOF as measured by the plasma membrane bystander to that of the direct complementation showed a strong positive correlation (Pearson correlation $=0.88$ ), suggesting that $\beta$-arrestin recruitment measured through the plasma membrane bystander was indeed a faithful metric (Figure S1D, right). The most extreme outlier, the serotonin $2 \mathrm{C}$ receptor (HTR2C), showed $\beta$ arr2 recruitment is PIP-binding independent as measured by the direct recruitment assay, but partially PIP-bindingdependent when measured using the plasma membrane bystander. Interestingly, this receptor was found to recruit arrestin even when PIP2 was acutely depleted (Toth et al., 2012). Additional receptors found to exhibit reduced PIP-binding sensitivity in the direct recruitment assay, for ßarr2, included $\alpha 1 B A R$ and $\beta 1$ AR, both of which exhibit some level of Gq coupling (Inoue et al., 2019). We speculate for cluster 1 receptors, such as HTR2C, which are primarily Gq-coupled, that their dependence on PIP-binding for arrestin recruitment to the plasma membrane may be amplified due to local PIP2-depletion upon stimulation.

We also asked whether $3 Q$ arrestins were differentially co-trafficked to endosomes. We used the FYVE domain of endofin as an endosome bystander (Endo) which we fused to LgBiT to monitored recruitment of arrestin bearing an $\mathrm{N}$-terminal SmBiT (Figure S1E), as was done for plasma membrane recruitment. Since both $\beta$ arr1 and $\beta$ arr2 displayed largely similar behavior in our plasma membrane recruitment assay, we focused on ßarr1 for these experiments; however, we also examined ßarr2 recruitment for selected receptors (Supplementary Data Figure 5B). All receptors known to mediate co-trafficking of arrestin to endosomes did so (Figure S1E, Supplementary data figure 5A-B), including OXTR, which showed measurable endosomal association of $\beta$ arr2, compared to weak and barely measurable $\beta$ arr1 endosome recruitment (Supplementary data figure 5C). In contrast, HTR2C showed more robust recruitment of $\beta$ arr1 than Barr2 (Supplementary Data Figure 5D). As expected, other cluster 1 receptors whose ability to co-traffic $\beta$-arrestins to endosomes had not yet been described displayed little signal for endosomal translocation, while other cluster 2 receptors showed robust signal for recruitment of both WT and $3 Q \beta$-arrestins.

Though end-point recruitment of $\beta$ arr1 to NTSR1, and other cluster 2 GPCRs was largely unaffected by loss of the PIP-binding site, prior NTSR1 experiments had found that loss of PIP binding slowed the kinetics of $\beta$-arrestin recruitment (Huang et al., 2020), suggesting PIP2 may play a role in the complexes formed with cluster 2 receptors, even when end-point recruitment is unchanged. We fit the rate of $\beta$-arrestin translocation to the plasma membrane in response to stimulation for all GPCRs in cluster 2 using our CAAX bystander NanoBiT assay (Figure 1B, top). 
As was seen for NTSR1, other cluster 2 GPCRs showed a slower association for $3 \mathrm{Q}$ than WT (Figure S2A). Though the magnitude of the effect varied across receptors (Figure S2B), these results clearly show that even recruitment to cluster 2 GPCRs is impacted by loss of PIP-binding in $\beta$-arrestins.

Together, these results provide several major findings: the first being that, generally, GPCRs that co-traffic with $\beta$-arrestins to endosomes do not require the PIP-binding capacity of $\beta$-arrestins for plasma membrane recruitment and are henceforth referred to as PIP-independent GPCRs. Secondly, though PIP-independent GPCRs retained the ability to recruit $\beta$-arrestins, the kinetics of recruitment were generally impaired by loss of PIP binding, suggesting that PIP-mediated interactions still contributed to recruitment for these receptors. Finally, while most GPCRs showed similar behavior between $\beta$ arr1 and $\beta$ arr2, there were exceptions for recruitment and PIP-bindingdependence, which supports the notion that there are receptor specific recruitment properties that remain poorly understood.

\section{Phosphorylation sites dictate dependence on PIP-binding}

As the distinction between class $A$ and class $B$ receptors was previously attributed to the presence of suitably positioned clusters of phosphosites in the receptor C-terminus (Oakley et al., 2001), we reasoned that there must be a degree of phosphorylation required to overcome the dependence on arrestin-PIP binding for recruitment to class A receptors. We chose the NTSR1 as a model receptor since WT NTSR1 stably associated with arrestins and the major phosphorylation cluster responsible for this phenotype were previously established for the rat ortholog (Oakley et al., 2001). Using human NTSR1, we designed a set of phosphorylationdeficient mutants, including both the C-terminus and the third intracellular loop (ICL3) (Figure 2A). ICL3 was found to be subject phosphorylation and appeared to make contacts to arrestin in a recent structure of NTSR1- $\beta$ arr1 (Huang et al., 2020), though the role of ICL3 phosphorylation in arrestin recruitment had not previously been explored. NTSR1 contains four S/T residues in ICL3, three of which are clustered together, and $9 \mathrm{~S} / \mathrm{T}$ residues in its C-terminus, 6 of which are divided into two clusters. We compared the PIP-dependence of phosphorylation mutants (Figure 2A) for recruitment of $\beta$ arr1 to the plasma membrane, using the previously described NanoBiT assay (Figure 1A). We first measured surface expression of the NTSR1 constructs and observed similar expression levels (Figure S3A), with the exception of ICL3-4A, which showed somewhat reduced expression. In addition, we confirmed that at the level of expression used the NanoBiT response was saturated making differences in amplitude unlikely to arise from any slight variations in expression between constructs (Figure S3B-C). Though WT NTSR1 was classified as a PIPindependent receptor, NTSR1 phosphorylation mutants could either be classified into both cluster 1 or cluster 2 (Figure 2A, Supplementary Data Figure 6), suggesting that particular phosphorylation mutants rendered arrestin recruitment to NTSR1 PIP-dependent. Removal of the two C-terminal phosphorylation site clusters (NTSR1-6A, NTSR1-10A) resulted in a dramatic reduction in arrestin recruitment (Supplementary Data Figure 6A), with remaining arrestin recruitment being largely PIP-dependent. Removal of the ICL3 phosphorylation sites did not affect PIP-dependence (NTSR1-ICL3-4A), and neither did removal of the proximal phosphorylation cluster (NTSR1-AVAA), nor did removal of any one residue in the distal cluster (NTSR1-TLSA, NTSR1-ALSS, NTSR1-TLAS). However, removal of the distal phosphorylation cluster (NTSR1ALAA) led to a dramatic reduction in recruitment, and an increase in PIP-dependence, consistent with findings that the distal cluster in the rat ortholog is necessary for stable arrestin association (Oakley et al., 2001). NTSR1-5A, bearing a single C-terminal phosphorylation site in the distal cluster, showed PIP sensitivity comparable to NTSR-ALAA, while NTSR1-4A with two distal cluster phosphorylation sites showing much less PIP-dependence, suggesting that two 
301

302

303

304

305

306

phosphorylation sites are sufficient to overcome the need for PIP binding. Similarly, NTSR1TLAA, which differs from NTSR1-5A only in the addition of the proximal cluster of phosphosites exhibits sensitivity between the NTSR $1-5 A$ and NTSR1-4A constructs, suggesting that a phosphorylation site from the proximal cluster may offer a partial rescue for the absence of one in the distal cluster.

As the plasma membrane bystander recruitment assay suggested that two phosphorylation sites were necessary to overcome the PIP-dependence on arrestin recruitment, we wondered whether this behavior coincided with an ability to co-traffic arrestin to endosomes. We monitored translocation of arrestin to endosomes using the endosome bystander NanoBiT assay (Figure S1E). As expected, NTSR1-ALAA (Oakley et al., 2001) as well as NTSR1-6A and NTSR1-10A failed to recruit arrestin to endosomes (Figure 2B, Supplementary Data Figure 6B). A single Cterminal phosphorylation site (NTSR1-5A) was insufficient to promote arrestin traffic to endosomes; however, two phosphorylation sites in the distal cluster (NTSR1-4A) were sufficient to promote endosomal translocation. There was a further increase in recruitment when the proximal sites were returned (NTSR1-TLSA), suggesting an additional contribution from this region strengthens the interaction between NTSR1 and arrestin. Further support for a contribution from the proximal cluster stems from the difference between NTSR1-5A and NTSR1-TLAA, which differ in whether or not the proximal phosphorylation cluster is present and show a marked difference in both targeting of arrestin to endosomes, as well as PIP-dependence (Figure 2B). Within the distal cluster, any two phosphorylation sites were sufficient, and having the third present appeared to offer no additional benefit (NTSR1-AVAA compared to NTSR1-ALSS, NTSR1-TLAS and NTSR1-TLSA) (Figure 2B).

Given that two phosphorylation sites in the distal cluster were sufficient for both PIP-insensitivity for plasma membrane recruitment, and co-trafficking of arrestin to endosomes, we asked whether two phosphorylation sites were also sufficient for receptor internalization. We measured internalization of the NTSR1 constructs in $\Delta$ Barr1/2 HEK293 cells where either WT or 3Q $\beta$ arr1 was reintroduced. WT NTSR1 was robustly internalized by both WT and $3 Q$ Barr1. In contrast, NTSR1-5A showed a significant difference in internalization between WT and 3Q $\beta$ arr1, while NTSR1-4A showed no difference in internalization between WT and 3Q $\beta$ arr1. The trend between NTSR1-5A and NTSR1-4A parallels that seen for $\beta 2 A R$ and $\beta 2 A R-V 2 C$ (Figure 2C), supporting that two phosphorylation sites are sufficient for robust internalization that is PIP-independent. In addition, the internalization observed for NTSR1-5A by WT $\beta$ arr1 suggests that the lack of endosome recruitment observed for this construct (Figure 2B) is due to weakened GPCR-ßarr interaction and not simply a lack of internalization for this receptor.

Together, these data show that two suitably positioned phosphorylation sites are sufficient to render $\beta$-arrestin recruitment PIP-independent and allow for robust arrestin-dependent internalization as well as support co-trafficking of arrestin to endosomes. Furthermore, they show that NTSR1, a receptor that recruits $\beta$-arrestin in a PIP-independent manner, can become PIPdependent by changes in receptor phosphorylation.

\section{PIP2 binding affects complex stability and tail-core equilibrium in vitro}

As PIP-binding was previously suggested to stabilize the interaction between a GPCR and arrestin (Gaidarov et al., 1999), based on experiments in cells, we wanted to explicitly test this in vitro. Using NTSR1 as our model receptor, where PIP-binding was not strictly necessary for recruitment in cells, we compared the ability of GRK5 phosphorylated NTSR1 to form a complex with $\beta$ arr1 (WT or $3 \mathrm{Q}$ mutant) in the presence of a soluble PIP2 derivative, diC8-PI $(4,5) \mathrm{P} 2$ 
(henceforth PIP2), by size-exclusion chromatography (Figure 3A-B) (Huang et al., 2020). While

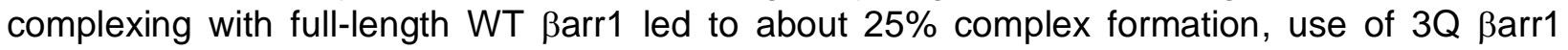
resulted in $<5 \%$ complex formation (Figure $3 C$ ). Use of a C-terminally truncated Barr1 (1-382) led to a more than 2-fold enhancement in complex formation, which was only slightly reduced with the corresponding $3 \mathrm{Q}$ arrestin. Using the LOF metric developed to evaluate the impact of PIPbinding on arrestin recruitment in cells, we found that full length arrestin showed a greater degree of LOF than C-terminally truncated arrestin, suggesting that removal of the arrestin C-tail is largely able to overcome the impairment in complexing that results from the $3 Q$ mutation (Figure S4A). Since arrestin activation is understood to proceed via initial release of its auto-inhibitory C-tail (Sente et al., 2018; Shukla et al., 2013), we wanted to rule-out the possibility that 3Q Barr1 complexing efficiency is simply reduced due to a lack of arrestin C-tail release. We designed a Förster Resonance Energy Transfer (FRET) sensor to report on arrestin C-tail release (Figure S4B): using a cysteine-free $\beta$ arr1 construct, we introduced two new cysteine residues at positions 12 and 387 - $\beta$ arr1 (12-387) - to allow for selective labeling of these positions with a suitable dye pair. Given that the expected change in distance between the bound and unbound C-tail was $\sim 40$ $\AA$ (Chen et al., 2017; Kim et al., 2012; Zhuo et al., 2014), we used an AlexaFluor 488/Atto 647N FRET pair, which offers a relatively short Förster radius $\left(R_{0} \sim 50 \AA\right)$. We found that GRK5phosphorylated NTSR1 robustly displaced the C-tail of both WT and 3Q Barr1 (12-387), comparably to that of a saturating concentration of phosphopeptide corresponding to the phosphorylated C-terminus of the vasopressin 2 receptor (henceforth V2Rpp) known to displace the arrestin C-tail (Shukla et al., 2013), even at concentrations 10x lower (Figure S4C). These data show that not only does in vitro phosphorylated NTSR1 fully displace the arrestin C-tail, but with higher efficacy than an equimolar concentration of phosphopeptide (even in the presence of unphosphorylated NTSR1), and this is independent of the PIP-binding ability of arrestin.

We reasoned that the reduced complexing efficiency of $3 Q \beta a r r 1$ may be due to differences in the proportion of core-engaged complex being formed. To test this hypothesis, we used an environmentally sensitive bimane fluorophore (BIM) site-specifically installed at L68 (L68BIM) on the arrestin finger loop, a region that upon formation of a core-engaged complex with an active GPCR becomes buried within the receptor TM core. Such a sensor had previously been used to report on core-engagement for rhodopsin/arrestin-1 (Sommer et al., 2005, 2006), where upon core-engagement a blue-shift and an increase in fluorescence emission occurs, owing to the bimane probe moving into a lower polarity environment within the receptor TM core.

While addition of V2Rpp to $\beta$ arr1 L68BIM leads to C-tail release and a $\sim 50 \%$ increase in bimane fluorescence as seen previously (Latorraca et al., 2020), we speculated that the addition of receptor may further increase this signal. We compared the fluorescence changes of $\beta$ arr1 L68bim (WT or 3Q) upon addition of NTSR1 that was either dephosphorylated or phosphorylated by GRK5 (Figure 3E). In the absence of phosphorylation, there was no increase in fluorescence; however, phosphorylated NTSR1 led to a 2-fold enhancement in fluorescence intensity. The addition of V2Rpp at a saturating concentration to the unphosphorylated NTSR1 did not result in a significant increase over phosphopeptide alone, consistent with the behavior observed for Ctail release (Figure S4C). Furthermore, this effect was only seen for WT ßarr1 L68BIM, and not the $3 \mathrm{Q}$ mutant, which showed an increase in fluorescence when V2Rpp was added, but no further enhancement with both unphosphorylated and GRK5 phosphorylated NTSR1.

We reason that if the complex exists as a dynamic equilibrium between three states (Figure 3F): dissociated, tail-bound and core-engaged. Then if PIP-binding serves to stabilize the coreengaged state loss of lipid binding would bias the equilibrium towards a tail-engaged state, which should have a similar spectroscopic signature to V2Rpp alone. Taken together, these data 
401

402

403

404

405

406

407

408

409

410

411

412

413

414

415

416

417

418

419

420

421

422

423

424

425

426

427

428

429

430

431

432

433

434

435

436

437

438

439

440

441

442

443

444

445

446

447

448

449

450

suggest a model of complex assembly where release of the arrestin C-terminus by the phosphorylated GPCR C-terminus is rapid, and reversible. The resulting tail-bound state is in equilibrium with a core-engaged state, where arrestin-lipid binding stabilized this state and thereby slowing dissociation. In the context of full length arrestin, destabilization of core-engaged state in the $3 Q$ mutant leads to a reduction in complex stability, presumably due to arrestin C-tailmediated dissociation from the tail-bound state. However, when the arrestin C-terminus is removed, the reduced core-engagement of the $3 Q$ mutant does not impact complexing efficiency due to the increased stability of the tail-bound state (as seen in Figure 3C, S4A).

\section{PIP2, in the absence of a GPCR, triggers conformational changes in arrestin}

Some GPCRs, such as the $\beta 1 A R, \beta 2 A R$ and D2R recruit arrestin to the plasma membrane in super-stoichiometric quantities, and without the need of a phosphorylated C-terminus, but this recruitment depends on the ability of arrestin to bind PIPs (Eichel et al., 2018)). Having shown that PIP-binding affects the dynamics of NTSR1- $\beta$ arr1 complexes in vitro, we wondered whether PIPs in the absence of an associated GPCR could also affect the conformation of Barr1. We compared the effect of PIP2 to the V2Rpp for promoting conformational changes in arrestin using FRET and fluorescence reporters on the finger loop, gate loop, and C-tail (Figure 4A).

Both the finger loop (Figure 4B, Figure S5A-B) and gate loop (Figure 4C, Figure S5C-D) showed saturable conformational changes upon addition of PIP2 which were smaller than those seen for V2Rpp. Further, the corresponding PIP-binding defective $3 \mathrm{Q}$ mutants did not show PIP2-induced conformational changes, though they responded to V2Rpp similarly to WT protein (Figure S5). These data suggest that binding of PIP2 to the arrestin C-lobe allosterically promotes conformational changes in key arrestin regions involved in GPCR recognition and activation. As the accepted mechanism for arrestin activation begins with release of its autoinhibitory C-tail (Sente et al., 2018), we wondered whether these conformational changes were the result of allosterically promoted C-tail release. Using our ßarr1 C-tail FRET sensor (Figure S4B) we found that PIP2 indeed promoted a small movement of the arrestin C-terminus (Figure 4D), but only at concentrations higher than those needed to saturate the responses seen for either the finger or gate loop sensors (Figure 4B-C). As was the case for the other sensors, this FRET change in response to PIP2 is absent in the corresponding $3 Q$ mutant (Figure S5E-F). This finding is consistent with recent DEER experiments that found little or no C-terminal displacement for ßarr1 with IP6 (Chen et al., 2021).

We reason that the conformational changes in the finger and gate loops observed together with the small FRET change in response to PIP2 could either be due to a change in the equilibrium of active-inactive $\beta$ arr1, or a population of an intermediate state of arrestin bearing a change in position or orientation of the arrestin C-tail within the arrestin $\mathrm{N}$-lobe.

\section{PIP2 increases the population of active arrestin}

While our fluorescence experiments support PIP2-promoted conformational changes consistent with arrestin activation, the lack of $\mathrm{C}$-tail release raised questions of whether these conformational changes truly reflected an increase in the population of active arrestin, as would be detected by arrestin binding partners. While the active form of arrestin is understood to mediate signaling via interactions through a number of protein partners, including MAPK, ERK, SRC (Ranjan et al., 2017; Reiter et al., 2012), there has been speculation that the binding of a particular partner might be mediated by a distinct arrestin conformation. We reasoned that the most objective way to probe the global activation state of arrestin was through the use of an engineered Fab (Fab30), which 
was raised to bind to the active (V2Rpp-bound) state of $\beta$ arr1 with high-affinity (Shukla et al., 2013). Fab30 has found utility in a number of structural studies (Lee et al., 2020; Nguyen et al., 2019; Shukla et al., 2013; Shukla et al., 2014; Staus et al., 2020), functional studies (Cahill et al., 2017; Ghosh et al., 2019; Kumari et al., 2016; Latorraca et al., 2020; Thomsen et al., 2016) and more recently it has been adapted as a single-chain intrabody (IB30) for the detection of active Barr1 in cells (Baidya et al., 2020a; Baidya et al., 2020b).

We used Surface Plasmon Resonance (SPR) to measure binding of Fab30 to immobilized $\beta$ arr 1 (Figure 5A). To confirm the immobilized arrestins behave as expected, we tested binding of V2Rpp and Fab30+V2Rpp (Figure 5B). Though selected for binding to the V2Rpp-bound state of $\beta$ arr1, Fab30 also bound to unliganded $\beta$ arr1 weakly, and interestingly this binding was enhanced when Fab30 was injected together with PIP2 (Figure 5B). This suggested that PIP2 increased the proportion of arrestin that can be stabilized in the active by Fab30, consistent with our fluorescence experiments that supported PIP2 playing a role in arrestin activation. We then compared the effect of PIP2 to that of PG, and PI(3)P for WT arrestin, but also the PIP-binding deficient 3Q mutant, and the pre-activated (1-382) arrestin (Kim et al., 2013). At $1 \mu \mathrm{M}$, Fab30 alone showed $10.2 \pm 0.9 \%$ (of maximal) binding to WT Barr1, compared to $56.8 \pm 2.0 \%$ binding for the C-terminally truncated arrestin (Figure $5 \mathrm{C}$ ). This suggests that Fab30 binding is favored by a conformation accessible to WT arrestin, but greatly enhanced by removal of the arrestin Cterminus. When Fab30 is injected together with a saturating concentration of PIP2 $(40 \mu \mathrm{M})$, binding to WT arrestin increased more than 3 -fold, to $33.9 \pm 1.8 \%$, compared to Fab30 alone. In contrast, $\mathrm{PI}(4,5) \mathrm{P} 2$ had a smaller effect on the pre-activated (1-382) arrestin, but still increased binding from $56.8 \%$ to $65.9 \pm 0.8 \%$. In the case of the $3 Q$ mutant, PIP2 still enhanced binding of Fab30, but significantly less than for WT. PG and PI(3)P enhanced binding of Fab30 to WT arrestin, relative to unliganded, though the effect was small, and more specifically was significantly less than that seen with $\mathrm{PI}(4,5) \mathrm{P} 2$. Further, in the case of $3 \mathrm{Q}$ arrestin, there was no difference between $\mathrm{PG}, \mathrm{PI}(3) \mathrm{P}$ and $\mathrm{PI}(4,5) \mathrm{P} 2$, suggesting that while any anionic lipids may weakly increase Fab30 binding to arrestin, $\mathrm{PI}(4,5) \mathrm{P} 2$ affected a specific increase in Fab30 binding. Both PG and PI(3)P did not enhance Fab30 binding to 1-382 $\beta$ arr1.

Based on these data we propose that spontaneous activation of arrestin to an active-state capable of binding Fab30 is possibly but rare in the absence of arrestin inputs (Figure 5D). V2Rpp is able to dramatically shift the equilibrium towards the active-state by displacement of the arrestin Cterminus, and removal of the arrestin C-terminus alone is sufficient to greatly enhance the activepopulation, even in the absence of any arrestin ligand. Unlike V2Rpp, which displaces the arrestin C-terminus, PIP2 which is not able to displace the arrestin C-terminus is only able to partially stabilize the active-state of arrestin. Alternatively, PIP2 stabilizes a distinct intermediately-active arrestin capable of binding Fab30, although with a lower affinity.

\section{Conclusions:}

Our results reveal new molecular details underpinning the regulation of arrestin recruitment to GPCRs, and how spatial and temporal control of GPCR- $\beta$-arrestin complexes may occur within a cell.

In particular, these findings offer a molecular basis for understanding the phenotypic classification of GPCRs as "class A" or "class B". In our model (Figure 6), we refer to "class A" and "class B" GPCRs as "PIP-dependent" and "PIP-independent", respectively. "PIP-dependent" GPCRs (Figure 6, left) require the coincident detection of membrane PIPs together with the activated and phosphorylated GPCR for arrestin recruitment. We speculate that this may be due to an 
501

502

503

504

505

506

507

508

insufficiency in phosphorylation of these receptors, requiring either an allosteric priming of C-tail release by plasma membrane PIPs, such as $\mathrm{PI}(4,5) \mathrm{P} 2$, or that both phosphate-mediated contacts and PIP-mediated contacts are required to act in concert to form a sufficiently long-lived complex for normal function. As some PIP-dependent GPCRs can recruit arrestin in a C-terminusindependent manner, this may suggest that release of the arrestin C-terminus may not be necessary for arrestin function in the context of these receptors. A further trait of these PIPdependent GPCRs is that they exhibit, to a varying degree, the "catalytic activation" phenotype (Eichel et al., 2018) wherein arrestin, after recruitment to an active GPCR, loses association with the GPCR but remains at the plasma membrane and concentrates at CCSs. This can be explained by the increasing concentration gradient of PIP2 leading into the CCS (Sun et al., 2007), together with our biophysical evidence that arrestin is not only able bind PIP2, but that PIP2 is able to promote conformational transitions associated with activation. Once a GPCR cargo has been translocated into a CCS, clathrin-mediated endocytosis (CME) proceeds and PIP2 levels drop. We believe that this may serve as the timing component for arrestin dissociation from these PIP-dependent GPCRs. Presumably, once arrestin has dissociated, the receptor is susceptible to dephosphorylation, and upon arrival at early endosomes is able to be sorted for rapid recycling (Krueger et al., 1997). In contrast, "PIP-independent receptors" (Figure 6, right panel) possess phosphorylation sites which alone are able to promote a stable association with arrestin, without the need for membrane PIPs. Since PIP-binding is not necessary to maintain the GPCR-arrestin association, arrestin is able to co-traffic with these receptors to endosomes.

These data suggest that while PIP-mediated contacts are not necessary to maintain association, they likely affect the equilibrium of core vs. tail-engaged states of the complex. We speculate that this shift in equilibrium, particularly in the context of endosomes defined by $\mathrm{PI}(3) \mathrm{P}$, may explain how PIP-independent receptors, such as V2R and PTH1R are able to engage both $\beta$-arrestin and G proteins simultaneously in a so-called "megaplex" assembly (Nguyen et al., 2019; Thomsen et al., 2016).

To-date four structures of GPCR- $\beta$ arr 1 complexes have been described, all of which show arrestin in a core-engaged state (Huang et al., 2020; Lee et al., 2020; Staus et al., 2020; Yin et al., 2019), but only one had PIP2 bound at the interface (Huang et al., 2020). Interestingly, this NTSR1-ßarr1 complex with PIP2 bound used the native NTSR1 C-terminus and did not use Fab30 to stabilize the complex. We speculate that Fab30 may play a particularly important role as previously it had been shown that Fab30 was essential for stabilizing the $\beta 2 A R-V 2 C-\beta a r r 1$ complex Fab30 (Shukla et al., 2014).

Overall, our data offer a parsimonious explanation for several phenotypic behaviors observed for GPCR- $\beta$-arrestin complexes and provide a biophysical framework to understand the interplay between phosphorylation-mediated and PIP-mediated contacts in complex assembly. A reliance on phosphoinositides for arrestin recruitment offers a robust solution for recruitment of arrestin to receptors with spatial control, and temporal precision. Given the interplay between PIP-dependent recruitment and phosphorylation, we believe that distinct signaling outcomes may not only be due to differences in phosphorylation alone (Latorraca et al., 2020), but rather that these differences may be further fine-tuned by membrane PIPs that are present in distinct subcellular locations, adding yet another layer of complexity to our understanding of GPCR signaling.

\section{Acknowledgements:}

We thank Betsy White (Stanford University) for technical laboratory assistance; Shoji Maeda (Stanford University) for providing purified Fab30 protein; Weijiao Huang (Stanford University) for 
551

552

553

554

555

556

557

558

559

560

561

562

563

564

565

566

567

568

569

570

571

572

573

574

575

576

577

578

579

580

581

582

583

584

585

586

587

588

589

590

591

592

593

594

595

596

597

598

599

600

601

assistance with NTSR1 expression and purification; Daniel Hilger (Stanford University) for purified BirA enzyme; Yoon Seok Kim and Eamon Byrne (Stanford University) for assistance with FSEC experiments. We also thank Kayo Sato, Yuko Sugamura, Shigeko Nakano and Ayumi Inoue at Tohoku University for plasmid construction and cell-based GPCR assays. This work was supported in part by National Institutes of Health grants R01NS028471 (B.K.K.), R01 Al125320 (K.C.G), R01DA010711 and R01DA012864 (M.vZ.). Additional support to both K.C.G and B.K.K. is provided by the Mathers Foundation. B.K.K. is a Chan-Zuckerberg Biohub Investigator. J.J. is a Damon Runyon Fellow supported by the Damon Runyon Cancer Research Foundation (DRG2318-18). B.B.R. is a recipient of an American Heart Association Predoctoral Fellowship (19PRE34380570). F.M. H. is a recipient of a Marie Skłodowska-Curie Individual Fellowship from the European Union's Horizon 2020 research and innovation programme (grant agreements No. 844622) and an American Heart Association postdoctoral fellowship (19POST34380839). M.M. was supported by an American Heart Association postdoctoral fellowship (17POST33410958). A.I. was funded by the PRIME 19gm5910013, the LEAP 20gm0010004 and the BINDS JP20am0101095 from the Japan Agency for Medical Research and Development (AMED); KAKENHI $17 \mathrm{~K} 08264$ and $21 \mathrm{H} 04791$ from by the Japan Society for the Promotion of Science (JSPS); JST Moonshot Research and Development Program JPMJMS2023 from Japan Science and Technology Agency (JST); Daiichi Sankyo Foundation of Life Science; Takeda Science Foundation; Ono Medical Research Foundation (A.I.); The Uehara Memorial Foundation.

\section{Author Contributions:}

J.J and A.I. conceived the project and designed experiments and analysis. J.J. expressed, purified and labeled proteins for biophysical measurements and performed in vitro experiments. R.K. and A.I. performed NanoBiT, flow-cytometry and western blot experiments. B.B-R. performed live-cell cAMP experiments overseen by M.vZ. J.J and D.H.S. performed SPR experiments, overseen by K.C.G. F.M.H. contributed to data analysis. M.M. and K.K. contributed to protein expression, purification and/or construct design. J.J. analyzed data with input from A.I., R.K., B.BR.,D.H.S.,F.M.H., M.vZ. and B.K.K. J.J. and B.K.K. wrote the paper with input from all authors.

\section{Declaration of Interests:}

B.K.K is a cofounder and consultant for conformetRx, Inc.

Figure titles and legends, tables with titles and legends:

Figure 1. Arrestin phosphoinositide binding is required for recruitment to some GPCRs A) cartoon depicting NanoBiT assay for measuring arrestin plasma membrane recruitment upon agonist stimulation. Upon complementation $\mathrm{SmBiT}$ and $\mathrm{LgBiT}$ form a functional NanoLuc luciferase. In key, "Phosphate" denotes phosphorylated Ser/Thr residues. B) Two representative GPCRs, $\beta 1 A R$ and NTSR1 illustrate data obtained for $\beta$-arrestin recruitment by NanoBiT assay shown in panel A. Data were collected over time after agonist addition ( $\mathrm{t}=0 \mathrm{~min}$ ), and values are shown as luminescence fold-change (over vehicle treatment) \pm standard deviation (measured as 2 technical replicates for each of $n=3$ independent experiments). Colors denote concentrations of agonist used for stimulation. Agonists used were isoproterenol for $\beta 1 A R$ and neurotensin for NTSR1. Grey boxes mark the time region (10-15 minutes post agonist addition) over which luminescence is integrated, for each concentration of agonist, to produce concentration response curves (bottom). WT and $3 Q$ amplitudes were determined as the difference of fitted pre- and posttransition plateaus. C) Plot of LOF values for panel of tested GPCRs. Points represent LOF value obtained as ratio of WT and $3 Q$ recruitment, and error bars reflect error in LOF derived from standard errors of fits (see methods). Dashed ellipses denote clusters obtained from $\mathrm{k}$ means 
602

603

604

605

606

607

608

609

610

611

612

613

614

615

616

617

618

619

620

621

622

623

624

625

626

627

628

629

630

631

632

633

634

635

clustering of data (see methods). Vertical grey lines denote $L O F=0$ and LOF $=1$; vertical purple and orange lines reflect the centers of the respective clusters from $\mathrm{k}$ means and correspond to $\mathrm{LOF}=0.06$ and LOF $=0.73$, respectively.

Figure 2. Receptor phosphorylation patterns govern PIP-dependence for arrestin recruitment. A) Left, schematic of human NTSR1 showing motifs in receptor ICL3 and C-terminus that are subject to phosphorylation. Phosphorylation sites examined in this study are shown in red and numbered 1-10 (above). Residue numbers corresponding to the region of human NTSR1 are listed at the start and end of the shown sequences. Construct key shows possible phosphosites as empty boxes, which when mutated to alanine are filled with an "X". Plasma membrane recruitment of arrestin upon stimulation of cells expressing different NTSR1 constructs, measured using the NanoBiT assay described in Figure 1. Points represent LOF value obtained as ratio of WT and $3 \mathrm{Q}$ recruitment, and error bars represent standard error of fits (see methods). Points are colored based on cluster designation obtained from $\mathrm{k}$ means clustering of all receptor-arrestin recruitment data. B) Translocation of Barr1 to endosomes upon stimulation of cells expressing different NTSR1 constructs, measured using an endosome bystander NanoBiT assay, as described in Figure S1. Points represent recruitment (fold chance over basal upon stimulation) for WT and $3 \mathrm{Q}$ recruitment, denoted by circles and triangles, respectively. Points are based on data from $n=3$ biological experiments. Error bars represent standard error of fit used to determine recruitment. Points are colored based on the cluster assignment of that mutant. C) Internalization, measured by loss of cell-surface receptors upon agonist stimulation, for $\Delta \beta$ arr1/2 cells expressing NTSR1 or $\beta 2 A R$ constructs and transfected with arrestin constructs indicated. Values represent independent experiments $(n=5-10)$. Internalization by $3 Q$ Barr 1 and mock were compared to WT using a two-tailed paired t-test. ns: $p>0.05 ;{ }^{*}: p<=0.05 ;{ }^{* *}: p<=0.01 ;{ }^{* * *}: p<=0.001 ;{ }^{* * *}: p<=$ 0.0001 .

Figure 3. Lipid binding stabilizes core-engaged arrestin complexes. A) cartoon of complexing efficiency assay. Size-exclusion chromatography (SEC) resolves complex from components. B) Representative experiment showing SEC chromatograms with vertical dashed lines indicating free NTSR1, complex, and free arrestins. C) Complexing efficiency, for NTSR1 with indicated arrestins. Boxplots: center line, median; box range, 25-75th percentiles; whiskers denote minimum-maximum values. Individual points are shown ( $n=6$ independent experiments). Twotailed unpaired t-test used to compare conditions. ns: $p>0.05 ;{ }^{* \star *}: p<=0.0001$. D) Cartoon showing equilibrium of NTSR1-arrestin complex. Pink star denotes L68BIM probe used for experiment shown in panel E. E) Bimane spectra for L68BIM labeled Barr1 in complex with NTSR1. All NTSR1 samples contained diC8-PI(4,5)P2 (4.1 $\mu \mathrm{M})$ Boxplots: center line, median; box range, 25-75th percentiles; whiskers denote minimum-maximum values. Individual points are shown ( $\mathrm{n}=3$ independent experiments). V2Rpp-NTSR1 (GRK5p) and V2Rpp-NTSR1 (unphos) + V2Rpp were compared by two-tailed unpaired t-test. ns: $p>0.05 ;{ }^{*}: p<=0.05$. Apo indicates free arrestin without any ligand present; unphos indicates unphosphorylated receptor and GRK5p indicates GRK5-in vitro phosphorylated receptor. Spectra are normalized to apo $(100 \%)$ within each experiment and the fluorescence intensity at lambda max was used as the value. F) Free energy diagram illustrating how PIP-binding, by stabilizing the core-engaged state of the NTSR1-arrestin complex slows arrestin dissociation. Loss of the PIP-binding element of arrestin destabilizes the core-engaged state, shifting equilibrium towards the tail-engaged state leading to a higher degree of complex disassembly. Removal of the arrestin C-terminus stabilizes the complex in the tail-engaged state and reduces disassembly even when core-engaged complex is destabilized by lack of PIP-binding. 
Figure 4. PIP2 alone promotes conformational changes in arrestin, including C-tail movement, but not release. A) overlay of inactive (PDB: 1G4M) [grey] and active (PDB: 4JQI) [black] ßarr1. The $\mathrm{N}$ and $\mathrm{C}$ lobes of $\beta$ arr1 are indicated. Activation leads to reorganization of several loops, and the gate loop and finger loop are highlighted. Re-orientation of these loops from inactive (yellow) to active (green) can be monitored by site-specific fluorescence spectroscopy. In finger loop inset the sphere denotes $C_{\alpha}$ L68C which is labeled with BIM. In gate loop inset, the sphere denotes Ca L293C which is labeled with NBD. An installed W residue replacing L167 dynamically quenches 293NBD. B) Spectra of bimane labeled (L68C) Barr1 in response to V2Rpp and PIP2. Arrow indicates direction of spectral shift with increasing concentration. Values are mean $\pm S D$ $(\mathrm{n}=3$ independent experiments). Spectra were normalized to the apo condition within a given experiment. C) Spectra of NBD labeled (L167W-L293C) Barr1 in response to V2Rpp and PIP2. Arrow indicates direction of spectral shift with increasing concentration. Spectra shown are from a single experiment shown ( $n=1$ independent experiments). Spectra were normalized to the apo condition. D) Cartoon showing how FRET change is linked to C-tail release, left. Right, spectra of AF488/AT647N labeled (A12C-V387C) Barr1 in response to V2Rpp and PIP2. Arrow indicates direction of spectral shift with increasing concentration. Spectra were normalized via donor intensity within a given experiment. Data shown are for a representative experiment.

Figure 5. PIP2 enhances Fab30 binding to $\beta$ arr1. A) Cartoon of surface plasmon resonance (SPR) experiments, where $\beta$ arr1 is immobilized via $\mathrm{N}$-terminal biotinylation and a Fab30 binder is injected in the presence of absence of arrestin ligands, lipids or V2Rpp. B) Representative sensogram for SPR binding experiment. With WT Barr1 immobilized, Fab30 (1 $\mu \mathrm{M})$ was injected together with either no ligand, V2Rpp $(40 \mu \mathrm{M})$ or di-C8-PIP2 $(40 \mu \mathrm{M})$. The shown sensogram is representative of the outcome seen for independent experiments $(n=3)$ (see also panel C). C) Binding of Fab30 to immobilized arrestin constructs in the presence of different arrestin ligands. Maximum binding is defined based on normalization of the observed response to the amount of arrestin immobilized for each construct. Ligands di-C8-PG $(40 \mu \mathrm{M})$, di-C8-PI(3)P $(40 \mu \mathrm{M})$, di-C8$\mathrm{PI}(4,5) \mathrm{P} 2(40 \mu \mathrm{M})$ and V2Rpp $(40 \mu \mathrm{M})$ were mixed with Fab30 $(1 \mu \mathrm{M})$ and injected together. Points reflect independent measurements; open points represent the binding observed for the ligand in the absence of Fab30. Fab30 binding was compared using a two-tailed unpaired t-test. ns: $p>0.05 ;{ }^{*}: p<=0.05 ;{ }^{* *}: p<=0.01 ;{ }^{* *}: p<=0.001$.

Figure 6. Model for phosphoinositide regulation of GPCR- $\beta$-arrestin complex assembly and disassembly. GPCRs stratify into two groups with respect to the strength of their interaction with $\beta$-arrestins: one group requires an interaction between $\beta$-arrestin and PIP2 at the plasma membrane for recruitment (PIP-dependent), while the other does not (PIP-independent). In the case of PIP-dependent GPCRs, arrestin engagement is unstable and can result in dissociation of arrestin from the receptor, while maintaining an association with the plasma membrane (left panel). PIP2 is enriched at CCSs and in both cases complex assembly can occur. During endocytosis, PIP2 is depleted and for PIP-dependent GPCRs, the loss of this PIP2 contact may facilitate dissociation of arrestin thereby allowing for receptor recycling. In contrast, a PIPindependent GPCR will retain the interaction with arrestin even once PIP2 is depleted owing to the strong phosphorylation-dependent interactions; however, the full-engaged state of the complex is less stable in endosomes than at the plasma membrane, thereby allowing further $\mathrm{G}$ protein engagement to occur.

\section{Methods}

\section{Plasmids}


701

702

703

704

705

706

707

708

709

710

711

712

713

714

715

716

717

718

719

720

721

722

723

724

725

726

727

728

729

730

731

732

733

734

735

736

737

738

739

740

741

742

743

744

745

746

747

748

749

750

For cell-based assays, we used human, full-length GPCR plasmids cloned into the pCAGGS vector or the pcDNA3.1 vector derived from a previous study (Inoue et al., 2019). GPCR constructs were N-terminally FLAG epitope-tagged when they were intended to compare with cell surface expression levels. Specifically, NTSR1 was fused to the N-terminal FLAG epitope tag with a linker (MDYKDDDDKGTELGS; the FLAG epitope tag is underlined) and inserted into the pcDNA3.1 vector. $\beta 2 A R$ and $\mu O R$ were fused to the $N$-terminal FLAG epitope tag with a preceding HA-derived signal sequence and a flexible linker (MKTIIALSYIFCLVFADYKDDDDKGGSGGGGSGGSSSGGG) and inserted into the pCAGGS vector. Unless otherwise noted, other GPCR constructs were untagged. For the bystander NanoBiT-based $\beta$-arrestin assays, human full-length $\beta$-arrestin ( $\beta$-arrestin1 or 2 ; WT or $3 Q$ ) was $\mathrm{N}$-terminally SmBiT-fused with the flexible linker (MVTGYRLFEEILGGSGGGGSGGSSSGG; the $\mathrm{SmBiT}$ is underlined) and inserted into the pCAGGS vector (SmBiT- $\beta$-arrestin) (Baidya et al., 2020a). For the plasma membrane-localizing tag, LgBiT was C-terminally fused to the CAAX motif derived from human KRAS (SSSGGGKKKKKKSKTKCVIM) through the same flexible linker (LgBiT-CAAX). For the endosome-localizing tag, LgBiT was $\mathrm{N}$-terminally fused with the human Endofin FYVE domain (amino-acid regions Gln739-Lys806) again through the same flexible linker (Endo-LgBiT). For the direct NanoBiT-based $\beta$-arrestin assay, human full-length $\beta$-arrestin was $\mathrm{N}$-terminally LgBiT-fused with the same flexible linker and inserted into the pCAGGS vector (LgBiT- $\beta$-arrestin). GPCRs were C-terminally SmBiT-fused with the flexible linker (GGSGGGGSGGSSSGGVTGYRLFEEIL; the SmBiT is underlined) and inserted into the pCAGGS vector (GPCR-SmBiT).

\section{Peptides}

The V2Rpp peptide (ARGRpTPPpSLGPQDEpSCpTpTApSpSpSLAKDTSS) was obtained by custom peptide synthesis (Tufts University Core Facility). Fab30 was expressed and purified as previously described (Shukla et al., 2013). The concentration of V2Rpp stocks were determined by reaction with Ellman's reagent as previously described (Latorraca et al., 2020).

\section{NanoBiT- $\beta$-arrestin recruitment assays}

$\beta$-arrestin recruitment to the plasma membrane was measured by the bystander NanoBiT- $\beta$ arrestin assays using the SmBiT- $\beta$-arrestin and the LgBiT-CAAX constructs. HEK293A cells (Thermo Fisher Scientific) were seeded in a $6-\mathrm{cm}$ culture dish (Greiner Bio-One) at a concentration of $2 \times 10^{5}$ cells per $\mathrm{ml}(4 \mathrm{ml}$ per dish hereafter) in DMEM (Nissui Pharmaceutical) supplemented with 10\% FBS (Gibco), glutamine, penicillin, and streptomycin, one day before transfection. The transfection solution was prepared by combining $5 \mu$ of polyethylenimine solution $(1 \mathrm{mg} / \mathrm{ml})$ and a plasmid mixture consisting of $100 \mathrm{ng} \mathrm{SmBiT-} \beta$-arrestin, $500 \mathrm{ng}$ LgBiTCAAX and $200 \mathrm{ng}$ of a test GPCR construct in $200 \mu \mathrm{l}$ of Opti-MEM (Thermo Fisher Scientific). For the NTSR1 titration experiment, diluted volume of the FLAG-NTSR1 plasmid (13 ng to $200 \mathrm{ng}$ )

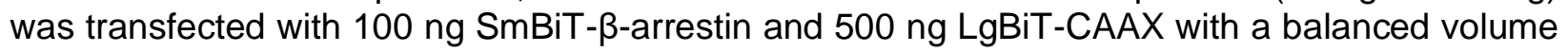
of the pcDNA3.1 vector (total plasmid volume of $800 \mathrm{ng}$ ). After an incubation for one day, the transfected cells were harvested with 0.5 mM EDTA-containing Dulbecco's PBS, centrifuged, and suspended in $2 \mathrm{ml}$ of Hank's balanced saline solution (HBSS) containing $0.01 \%$ bovine serum albumin (BSA fatty acid-free grade, SERVA) and 5 mM HEPES (pH 7.4) (assay buffer). The cell suspension was dispensed in a white 96-well plate (Greiner Bio-One) at a volume of $80 \mu \mathrm{l}$ per well and loaded with $20 \mu \mathrm{l}$ of $50 \mu \mathrm{M}$ coelenterazine (Carbosynth), diluted in the assay buffer. After $2 \mathrm{~h}$ incubation at room temperature, the plate was measured for its baseline luminescence (SpectraMax L, 2PMT model, Molecular Devices). Thereafter, $20 \mu \mathrm{l}$ of $6 \mathrm{X}$ ligand serially diluted in the assay buffer were manually added. The ligand used was dependent on the GPCR 
expressed, as described in Supplementary Data Table 1. The plate was immediately read for the second measurement as a kinetics mode and luminescence counts recorded for 15 min with an accumulation time of $0.18 \mathrm{sec}$ per read and an interval of $20 \mathrm{sec}$ per round. $\beta$-arrestin endosomal translocation was measured by following the same procedure as described above but using the $\mathrm{SmBiT}-\beta$-arrestin and the Endo-LgBiT constructs. Similarly, direct recruitment was measured by the same protocol as described above but using LgBiT- $\beta$-arrestin (500 ng) and C-terminally fusedSmBiT GPCR (500 ng) constructs. For every well, the recorded kinetics data were first normalized to the baseline luminescence counts.

\section{Analysis of cell-based recruitment data}

NanoBiT data were analyzed by converting kinetic data into concentration-response data by determining an average fold-change (relative to signal pre-stimulation) from 10-15 minutes postagonist addition. At least three independent experiments were performed for each receptorsensor combination. Concentration-dependent data from two technical replicates for each independent experiment were collectively fit to a four-parameter log logistic function (LL2.4) provided in the drc package of the statistical environment $\mathrm{R}$. This equation, of the form: $f(x)=$ $c+\frac{d-c}{1+e^{(b(\log (x)-\log (e)))}}$ provides pre- and post-transition values, $c$ and $\mathrm{d}$, respectively, that define the amplitude response for that assay. Cutoffs for bystander NanoBiT experiments were determined as based on a limit of detection of 3 s over the response of mock-transfected cells. Amplitude values were defined as amplitude $=$ top - bottom of fit, and amplitude error was calculated as $\delta($ amplitude $)=\sqrt{(\delta \text { top })^{2}+(\text { Sbottom })^{2}}$. Converting amplitude to LOF for each assay was based on the formula: 1 -amplitude $(3 Q) / \operatorname{amplitude}(W T)$. Errors for LOF were calculated as: $\delta(L O F)=L O F \sqrt{\left(\frac{\text { Samplitude }(3 Q)}{\text { amplitude }(3 Q)}\right)^{2}+\left(\frac{\text { Samplitude }(W T)}{\text { amplitude }(W T)}\right)^{2}}$. In cases where a fit failed to converge due to weak recruitment, these amplitudes and errors were set to zero. Recruitment of Barr1 (3Q) to $\mathrm{D} 1 \mathrm{R}$ in both plasma membrane bystander (CAAX) and direct recruitment which was set to zero. The error amplitude for ßarr1 (3Q) endosome translocation assay with D1R was also set to zero. The error amplitude for ßarr1 (3Q) endosome translocation assay with S1PR1 was set to zero, and the "top" value of the fit was set to 1.2 based on manual inspection. K means clustering was performed using pre-built functions in the tidyverse package of R. The number of clusters was varied from 1 to 10 and an elbow plot of within cluster sum of squares vs $k$ suggested 2 clusters fit the data well.

For recruitment kinetics, luminescence fold-change was plotted against time, and the values from zero to five minutes (initial rate) were fit to a logistic function of the form: $f(x)=\frac{L}{1+e^{-k\left(x-x_{0}\right)}}$, where $L$ is the curve's maximum value, $x_{0}$ is the value of the sigmoid midpoint and $k$ is the logistic growth rate. Fitting was done using the self-starting SSlogis four parameter nls function in the tidyverse package of $R$.

\section{GPCR internalization assay}

GPCR internalization assays was performed as described previously with minor modifications (Grundmann et al., 2018). $\Delta$ ßarr1/2 double knockout (DKO) cells, previously described (O'Hayre et al., 2017), were seeded in 6-cm dishes at concentration of $2 \times 10^{5} \mathrm{cells} / \mathrm{ml}$ (4 mL per dish) and cultured for 1 day before transfection. The cells were transfected with $1 \mu \mathrm{g}$ of the $\mathrm{N}$-terminally FLAG-tagged NTSR1 or the $\beta 2 A R$ construct, along with 200 ng of the WT or 3Q $\beta$ arr1 or empty plasmid, using PEI transfection reagent as described above. After 1-day culture, the transfected cells were harvested by EDTA-PBS and HEPES-HBSS and, following centrifugation, the cells 
were suspended in $500 \mu \mathrm{L}$ of $0.01 \%$ BSA-containing HEPES-HBSS. The cell suspension was dispensed in a 96-well V-bottom plate $(100 \mu \mathrm{L}$ per well) and mixed with $100 \mu \mathrm{L}$ of $2 \times$ GPCR solution ligand $(2 \mu \mathrm{M}$ neurotensin for FLAG-NTSR1 or $20 \mu \mathrm{M}$ Isoproterenol (Sigma-Aldrich) for FLAG-B2AR). After 30-min incubation in a $\mathrm{CO}_{2}$ incubator, the plate was centrifuged at $1,500 \mathrm{~g}$ for 5 min and the cells were washed twice with D-PBS. The cell pellets were suspended in $2 \%$ goat serum- and 2 mM EDTA-containing D-PBS (blocking buffer; $100 \mu \mathrm{L}$ per well) and incubated for 30 min on ice. After centrifugation, the cells were stained with anti-FLAG-epitope tag monoclonal antibody (Clone 1E6, FujiFilm Wako Pure Chemicals; $10 \mu \mathrm{g} \mathrm{mL}-1$ in the blocking buffer; $25 \mu \mathrm{L}$ per well) for $30 \mathrm{~min}$ on ice. After washing with D-PBS, the cells were labeled with a goat antimouse IgG secondary antibody conjugated with Alexa Fluor 647 (Thermo Fisher Scientific; $10 \mu \mathrm{g}$ $\mathrm{mL}^{-1}$ dilution in the blocking buffer; $25 \mu \mathrm{L}$ per well) for $15 \mathrm{~min}$ on ice. The cells were washed once with D-PBS, resuspended in $100 \mu \mathrm{L}$ of $2 \mathrm{mM}$ EDTA-containing-D-PBS and filtered through a 40 $\mu \mathrm{m}$ filter. The fluorescently labeled cells (approximately 20,000 cells per sample) were analyzed by the EC800 flow cytometer (Sony). Fluorescent signal derived from Alexa Fluor 647 was recorded in the FL3 channel. Mean fluorescence intensity (MFI) from all of the recorded events was analyzed by a FlowJo software (FlowJo) and used for statistical analysis.

\section{Cell-surface expression analysis by flow cytometry}

HEK293A cells were seeded in a 6 -well culture plate at concentration of $2 \times 10^{5}$ cells $/ \mathrm{ml}(2 \mathrm{~mL}$ per dish) and cultured for 1 day before transfection. The cells were transfected with $1 \mu \mathrm{g}$ of $\mathrm{N}$ terminally FLAG-tagged GPCR construct using PEI transfection reagent as described above and cultured for 1 day. The cells were collected by adding $200 \mu \mathrm{l}$ of $0.53 \mathrm{mM}$ EDTA-containing Dulbecco's PBS (D-PBS), followed by $200 \mu \mathrm{l}$ of $5 \mathrm{mM}$ HEPES (pH 7.4)-containing Hank's Balanced Salt Solution (HBSS). The cell suspension was transferred to a 96 -well V-bottom plate in duplicate and fluorescently labeled with the anti-FLAG epitope tag antibody and a goat antimouse IgG secondary antibody conjugated with Alexa Fluor 488 (Thermo Fisher Scientific, $10 \mu \mathrm{g}$ per $\mathrm{ml}$ diluted in the blocking buffer) as described above. Live cells were gated with a forward scatter (FS-Peak-Lin) cutoff at the 390 setting, with a gain value of 1.7 and fluorescent signal derived from Alexa Fluor 488 was recorded in the FL1 channel. For each experiment, the MFI value of mutants was normalized to that of WT performed in parallel.

\section{cAMP desensitization}

HEK293 $\triangle$ Barr1/2 (DKO) cells that endogenously express $\beta 2 A R$ were seeded into 6-well plates and transiently transfected after 24 hours with mApple, Barr2-mApple, or ßarr2(3Q)-mApple. Twenty-four hours after transfection, cells were transduced with CMV CADDis Green Upward CAMP sensor according to manufacturer instructions without addition of sodium butyrate (Montana Molecular \#U0200G) and seeded in triplicate in a black clear-bottom 96-well plate (Corning cat\# 3340). Twentyfour hours after transduction, the cells were washed once with $37^{\circ} \mathrm{C}$ assay buffer $[135 \mathrm{mM} \mathrm{NaCl}, 5$ $\mathrm{mM} \mathrm{KCl}, 0.4 \mathrm{mM} \mathrm{Mg} 2 \mathrm{Cl}, 1.8 \mathrm{mM} \mathrm{CaCl} 2,5 \mathrm{mM}$ glucose, $20 \mathrm{mM}$ HEPES pH 7.4], loaded into the prewarmed $37^{\circ} \mathrm{C}$ plate reader (Biotek Synergy $\mathrm{H} 4$ ), and equilibrated for five minutes. Prior to beginning the kinetic assay, mApple was read using monochromoters set to Ex:568/9.0 and Em:592/13.5. Then cADDis was read using monochromoters set to Ex:500/9.0 and Em:530/20.0. Three cADDis timepoints were collected to establish baseline, the plate was ejected, isoproterenol in $37^{\circ} \mathrm{C}$ assay buffer was added to a final concentration of $100 \mathrm{nM}$, and the plate was returned to continue collection. Thirty minutes after isoproterenol addition, 3-isobutyl-1-methylxanthine (IBMX) and forskolin (Fsk) in $37^{\circ} \mathrm{C}$ assay buffer were added to a final concentrations of $300 \mu \mathrm{M}$ and $10 \mu \mathrm{M}$ respectively. Responses were averaged across technical replicates, normalized to the maximum Fsk/IBMX response, and then 
848

averaged across independent experiments. Expression levels for cADDis and ßarr2 were normalized based on fluorescence.

\section{Western blotting}

HEK293A cells were transfected with the SmBiT- $\beta$-arrestin and the LgBiT-CAAX constructs by following the procedure described in the NanoBiT-based $\beta$-arrestin assay. After 1 -day culture, the transfected cells were lysed by SDS-PAGE sample buffer (62.5 mM Tris-HCl (pH 6.8), $50 \mathrm{mM}$ dithiothreitol, 2\% SDS, 10\% glycerol and $4 \mathrm{M}$ urea) containing $1 \mathrm{mM}$ EDTA and $1 \mathrm{mM}$ phenylmethylsulfonyl fluoride. Lysates derived from an equal number of cells were separated by $8 \%$ SDS-polyacrylamide gel electrophoresis. Subsequently, the proteins were transferred to PVDF membrane. The blotted membrane was blocked with $5 \%$ skim milk-containing blotting buffer (10 mM Tris-HCl (pH 7.4), $190 \mathrm{mM} \mathrm{NaCl}$ and 0.05\% Tween 20), immunoblot with primary ( $1 \mathrm{\mu g}$ per $\mathrm{mL}$, unless otherwise indicated) and secondary antibodies conjugated with horseradish peroxidase (1:2000 dilution). Primary antibodies used in this study were: anti- $\beta$-arrestin1 (rabbit monoclonal; CST, \#12697, D8O3J), anti- $\beta$-arrestin2 antibody (rabbit monoclonal; CST, \#3857, C16D9) and anti- $\alpha$-tubulin antibody (mouse monoclonal, clone DM1A; Santa Cruz Biotechnologies, sc-32293; 1:2000 dilution). Secondary antibodies were anti-rabbit IgG (GE Healthcare, NA9340) and anti-mouse IgG (GE Healthcare, NA9310). Membrane was soaked with an ImmunoStar Zeta reagent (FujiFilm Wako Pure Chemical). Chemiluminescence image of the membrane was acquired, and band intensity was analyzed with Amersham Imager 680 (Cytiva).

\section{NTSR1 expression and purification}

Full length human NTSR1 was modified with an N-terminal Flag tag followed by an octa-histidine tag and cloned into pFastBac1 vector. NTSR1 was expressed in Sf9 insect cells (Expression Systems) using a FastBac-derived baculovirus. Cells were infected at a density of $4 \times 10^{6} \mathrm{cells} / \mathrm{mL}$ and harvested $60 \mathrm{hrs}$ post infection. Cells were lysed in hypotonic buffer (10 mM HEPES, pH 7.4, and protease inhibitors) and solubilized at $4^{\circ} \mathrm{C}$ for 2 hours in a buffer containing $1 \%$ lauryl maltose neopentyl glycol (LMNG, Anatrace), $0.1 \%$ cholesteryl hemisuccinate tris salt (CHS, Steraloids), $0.3 \%$ sodium cholate (Sigma), $20 \mathrm{mM} \mathrm{HEPES} 7.4,500 \mathrm{mM} \mathrm{NaCl}, 25 \%$ glycerol, iodoacetamide (to cap cysteine residues) and protease inhibitors. Insoluble debris was removed by centrifugation and the supernatant was incubated with Ni-NTA (Qiagen) resin for 1 hour at $4{ }^{\circ} \mathrm{C}$. The resin was washed in batch with buffer containing $0.01 \%$ LMNG, $0.001 \%$ CHS, $0.003 \%$ sodium cholate, 20 $\mathrm{mM}$ HEPES $\mathrm{pH} 7.4,500 \mathrm{mM} \mathrm{NaCl}, 10 \mathrm{mM}$ imidazole and eluted with the same buffer supplemented with $200 \mathrm{mM}$ imidazole, $2 \mathrm{mM} \mathrm{CaCl}_{2}$ and $10 \mu \mathrm{M} \mathrm{NTS}_{8-13}$ (Acetate salt, Sigma). The eluate was loaded onto M1 FLAG immunoaffinity resin and washed with buffer containing $0.01 \%$ LMNG, 0.001\% CHS, 0.003\% sodium cholate, 20 mM HEPES pH 7.4, $500 \mathrm{mM} \mathrm{NaCl,} 10 \mathrm{mM}$ imidazole, $0.1 \mu \mathrm{M} \mathrm{NTS}_{8-13}$ and $2 \mathrm{mM} \mathrm{CaCl}_{2}$. The receptor was eluted with buffer containing 100 $\mathrm{mM} \mathrm{NaCl}, 20 \mathrm{mM}$ HEPES pH 7.4, 0.005\% LMNG, 0.005\% CHS, $1 \mu \mathrm{M} \mathrm{NTS}$ 8-13, $0.2 \mathrm{mg} / \mathrm{mL}$ flag peptide (DYKDDDDK) and $5 \mathrm{mM}$ EDTA. Elution fractions containing receptor were pooled and subjected to polishing by SEC on a Superdex 200 Increase 10/300 GL column (GE Healthcare) in $20 \mathrm{mM}$ HEPES, pH 7.4, $100 \mathrm{mM} \mathrm{NaCl}, 0.0025 \%$ LMNG, 0.00025\% CHS, and $0.1 \mu \mathrm{M} \mathrm{NTS}_{8-13}$. Peak fractions were pooled and concentrated to $200 \mu \mathrm{M}$ and aliquots were flash-frozen and stored at $-80^{\circ} \mathrm{C}$ until use.

\section{GRK5 expression and purification}

Full length human GRK5 was modified with a C-terminal hexa-histidine tag and cloned into pVL1392 vector for baculovirus production. GRK5 was expressed and purified as previously 
898

899

900

901

902

903

904

905

906

907

908

909

910

911

912

913

914

915

916

917

918

919

920

921

922

923

924

925

926

927

928

929

930

931

932

933

934

935

936

937

938

939

940

941

942

943

944

945

946

published (Beyett et al., 2019). Briefly, Sf9 insect cells (Expression Systems) were infected with a BestBac-derived baculovirus at a density of $3.5 \times 10^{6}$ cells $/ \mathrm{mL}$ and harvested 48 hours post infection. Cells were resuspended, lysed by sonication and the supernatant was applied to $\mathrm{Ni}$ NTA resin. The resin was washed with lysis buffer and GRK5 eluted with lysis buffer supplemented with $200 \mathrm{mM}$ imidazole. The combined eluate was then subjected to cationexchange chromatography using a MonoS 10/100 column (GE healthcare) and eluted with a linear gradient of $\mathrm{NaCl}$. Fractions containing GRK5 were combined and run on a Superdex 200 10/300 GL column (GE healthcare). GRK5 was aliquoted, flash frozen, and stored at $-80{ }^{\circ} \mathrm{C}$ until use.

\section{Arrestin expression and purification}

The parent construct for $\beta$-arrestin 1 ( $\beta$ arr1) is the long splice variant of human, cysteine-free (C59V, C125S, C140L, C150V, C242V, C251V, C269S) $\beta$-arrestin 1. This construct is modified with an N-terminal 6x Histidine tag, followed by a 3C protease site, a GG linker, AviTag and GGSGGS linker. The sequence was codon-optimized for expression in $E$. coli and cloned into a $\mathrm{pET}-15 \mathrm{~b}$ vector. Point mutations were prepared using site-directed mutagenesis. $\beta$-arrestin 1 (1382) was prepared by truncating $\beta$-arrestin 1 at residue 382 . All arrestin constructs used were prepared as follows: NiCo21(DE3) competent $E$. coli (NEB) were transformed, and large-scale cultures were grown in TB + ampicillin at $37^{\circ} \mathrm{C}$ until an $\mathrm{OD}_{600}$ of 1.0 . Cells were then transferred to room temperature and induced with $25 \mu \mathrm{M}$ IPTG when the $\mathrm{OD}_{600}$ reached 2.0. Cells were harvested $20 \mathrm{~h}$ post induction and resuspended in lysis buffer $(50 \mathrm{mM} \mathrm{Hepes} \mathrm{pH} \mathrm{7.4,500} \mathrm{mM}$ $\mathrm{NaCl}, 15 \%$ glycerol, $7.13 \mathrm{mM} \mathrm{BME}$ ) to a final volume of $40 \mathrm{~mL} / \mathrm{L}$ of cells. Cells were lysed by sonication and the clarified lysate applied to nickel sepharose and batch incubated for $1.5 \mathrm{~h}$ at 4 ${ }^{\circ} \mathrm{C}$. The resin was washed with 10 column volumes of wash buffer $(20 \mathrm{mM} \mathrm{HEPES} \mathrm{pH} \mathrm{7.4,} 500$ $\mathrm{mM} \mathrm{NaCl}, 10 \%$ glycerol, $7.13 \mathrm{mM} \mathrm{BME})+20 \mathrm{mM}$ imidazole, followed by 10 column volumes of wash buffer $+40 \mathrm{mM}$ imidazole. The protein was then eluted with 5 column volumes of wash buffer $+200 \mathrm{mM}$ imidazole and dialyzed overnight in $100 x$ volume of dialysis buffer $(20 \mathrm{mM}$ Hepes $7.4,200 \mathrm{mM} \mathrm{NaCl}, 2 \mathrm{mM} \mathrm{BME}, 10 \%$ glycerol) in the presence of 1:10 (w:w) of 3C protease. The digested protein was then subjected to reverse-Nickel purification and diluted with dialysis buffer containing no $\mathrm{NaCl}$ to bring the $\mathrm{NaCl}$ concentration to $75 \mathrm{mM}$. The protein was then purified by ion exchange chromatography (mono Q 10/100 GL, GE Healthcare), followed by SEC using a Superdex 200 increase 10/300 GL column (GE Healthcare) with SEC buffer (20 mM HEPES pH $7.4,300 \mathrm{mM} \mathrm{NaCl}, 10 \%$ glycerol). Purified protein was concentrated to between 100-300 $\mu \mathrm{M}$ using a $30 \mathrm{kDa}$ spin concentrator and aliquots were flash-frozen in liquid nitrogen and stored at $80{ }^{\circ} \mathrm{C}$ until use.

\section{Arrestin labeling and biotinylation}

Following SEC, elution peak fractions were pooled to a concentration of 10-20 $\mu \mathrm{M}$ and labeled with fluorophore(s): monobromobimane $(\mathrm{mBBr})$, Thermo Fisher Scientific M1378; N,N'-Dimethyl$\mathrm{N}$-(lodoacetyl)-N'-(7-Nitrobenz-2-Oxa-1,3-Diazol-4-yl)Ethylenediamine (IANBD amide), Thermo Fisher Scientific D2004; or a 1:3 mixture of Alexa Fluor 488 C5 Maleimide, Thermo Fisher Scientific A10254, and Atto647N Maleimide, ATTO TEC AD647N-41, respectively. Fluorophores were dissolved to in DMSO and added at 10x molar excess over protein, then allowed to react for $1 \mathrm{~h}$ at room temperature prior to quenching with cysteine (10x molar excess over fluorophore). The labeling reaction was further incubated for 10 minutes after cysteine addition, after which samples were spin filtered and subjected to a second round of size-exclusion chromatography, as detailed above, to remove free dye. The purified, was concentrated to between 100-300 $\mu \mathrm{M}$ 
using a $30 \mathrm{kDa}$ spin concentrator and aliquots were flash-frozen in liquid nitrogen and stored at $80^{\circ} \mathrm{C}$ until use.

Arrestins (SEC-pure) were biotinylated using recombinant BirA enzyme, according to commercial protocols (Avidity), with exception that biotinylation was carried out for $12 \mathrm{~h}$ at $4{ }^{\circ} \mathrm{C}$, rather than $30^{\circ} \mathrm{C}$. After biotinylation was complete, the reaction was flowed over $100 \mu \mathrm{L}$ (packed) of nickel Sepharose, equilibrated in arrestin SEC buffer and supplemented with $10 \mathrm{mM}$ imidazole, then washed with $200 \mu \mathrm{L}$ of the equilibration buffer. The combined flow-through and wash fractions were then purified by size-exclusion as described above.

\section{NTSR1 phosphorylation}

NTSR1 $(2.5 \mu \mathrm{M})$ was equilibrated in phosphorylation buffer $(20 \mathrm{mM}$ bis-tris propane (BTP) $\mathrm{pH}$ 7.5, $35 \mathrm{mM} \mathrm{NaCl}, 5 \mathrm{mM} \mathrm{MgCl}$, $20 \mu \mathrm{M} \mathrm{NTS}_{8-13}, 20 \mu \mathrm{M}$ C8-PI(4,5)P2, $0.05 \mathrm{mM}$ TCEP, 0.002\% MNG, $0.0002 \% \mathrm{CHS}$ ) at $25^{\circ} \mathrm{C}$ with gentle mixing for $1 \mathrm{~h}$. GRK5 was added to the reaction to a final concentration of $200 \mathrm{nM}$, and briefly incubated while the reaction was warmed from $25^{\circ} \mathrm{C}$ to $30{ }^{\circ} \mathrm{C}$. ATP was added to a final concentration of $1 \mathrm{mM}$. Upon completion, the reaction was supplemented with $\mathrm{CaCl}_{2}$ to a final concentration of $2 \mathrm{mM}$ and applied to an equilibrated M1 FLAG immunoaffinity resin and washed with buffer containing $0.004 \%$ LMNG, 0.004\% CHS, $20 \mathrm{mM}$ HEPES pH 7.4, $100 \mathrm{mM} \mathrm{NaCl}, 0.2 \mu \mathrm{M} \mathrm{NTS}_{8-13}, 2 \mathrm{mM} \mathrm{CaCl}_{2}$. The receptor was eluted with buffer containing $100 \mathrm{mM} \mathrm{NaCl}, 20 \mathrm{mM}$ HEPES pH 7.4, 0.004\% LMNG, 0.004\% CHS, $0.2 \mu \mathrm{M} \mathrm{NTS}_{8-13}$, $0.2 \mathrm{mg} / \mathrm{mL} 1 \times$ flag peptide (DYKDDDDK), $5 \mathrm{mM}$ EDTA), followed by SEC using a Superdex 200 increase 10/300 GL column (GE Healthcare) with SEC buffer (20 mM HEPES pH 7.4, $100 \mathrm{mM}$ $\mathrm{NaCl}, 0.004 \%$ LMNG, $0.0004 \% \mathrm{CHS})$.

\section{Analytical fluorescence-detection size-exclusion chromatography}

In a final volume of $20 \mu \mathrm{L}$, NTSR1 $(4.5 \mu \mathrm{M})$, the respective arrestin construct $(9 \mu \mathrm{M}), \mathrm{NTS}_{8-13}$ peptide $(50 \mu \mathrm{M})$ and diC8-PI $(4,5) \mathrm{P} 2(5 \mu \mathrm{M})$ were incubated in buffer containing $20 \mathrm{mM}$ HEPES $\mathrm{pH} 7.4,100 \mathrm{mM} \mathrm{NaCl}, 0.004 \%$ LMNG, $0.0004 \%$ CHS and $0.2 \mu \mathrm{M} \mathrm{NTS}_{8-13}$. Using a Prominence-i LC autosampler (Shimadzu), $10 \mu \mathrm{L}$ was injected onto a ENrich size-exclusion chromatography $65010 \times 300$ column (Bio-rad) pre-equilibrated in $20 \mathrm{mM} \mathrm{HEPES} \mathrm{pH} 7.4100 \mathrm{mM} \mathrm{NaCl}, 0.004 \%$ LMNG, $0.004 \% \mathrm{CHS}$ and $0.2 \mu \mathrm{M} \mathrm{NTS}{ }_{8-13}$, and run at a flow rate of $0.8 \mathrm{ml} / \mathrm{min}$. Tryptophan fluorescence was monitored at $\lambda(E X)$ of $280 \mathrm{~nm}$ and $\lambda(E M)$ of $340 \mathrm{~nm}$. Peaks in the obtained sizeexclusion chromatograms were modeled as gaussians, deconvolved and quantified (AUC) using Magic Plot 3 (Magic Plot).

\section{Surface plasmon resonance measurements}

SPR experiments were performed using a GE Biacore T100 instrument. Approximately 300-400 resonance units $(R U)$ of FPLC-purified biotinylated arrestin in HBS-P+ Buffer (GE Healthcare) were captured on an SA-chip (GE Healthcare), including a reference channel for online background subtraction of bulk solution refractive index and for evaluation of non-specific binding of analyte to the chip surface (Biacore T100 Control Software; GE Healthcare). All measurements were performed with 2 -fold serial dilutions using $60 \mathrm{~s}$ association followed by a dissociation time of more than $240 \mathrm{~s}$ at $25^{\circ} \mathrm{C}$ with a flow rate of at $30 \mu \mathrm{min}^{-1}$. Regeneration was performed by 2 injections of $2 \mathrm{M} \mathrm{MgCl}_{2}$ for $10 \mathrm{~s}$ at $50 \mu \mathrm{min}^{-1}$ flow rate. Single cycle measurements were performed as described above. All single cycle measurements were performed as triplicates and quantifications calculated to the $R U_{\max }$ of the individual immobilized ligands.

\section{Bulk fluorescence measurements}


Bulk fluorescence measurements were performed on either a Fluorolog instrument (Horiba) using FluorEssence v3.8 software and operating in photon-counting mode, or a Tecan Infinite M1000 PRO multimodal microplate reader (Tecan). Fluorolog measurements of bimane-labeled ßarr1 constructs (NTSR1 experiments) were performed at final concentration of $0.4 \mu \mathrm{M}$ [arrestin] in buffer containing $20 \mathrm{mM}$ HEPES pH 7.4, $100 \mathrm{mM} \mathrm{NaCl}$ and $0.004 \%$ LMNG (w/v)/0.0004\% CHS

1003

1004

1005

1006

1007

1008

1009

1010

1011

1012

1013

1014

1015

1016

1017

1018 (w/v) supplemented with $4 \mu \mathrm{M}$ NTS(8-13). For NTSR1 experiments the following concentrations were used: $4 \mu \mathrm{M}$ NTSR1, $4.1 \mu \mathrm{M}$ diC8-PI(4,5)P2, $50 \mu \mathrm{M}$ V2Rpp (depending on condition). Samples were incubated for $1 \mathrm{~h}$ in the dark before measurement. Fluorescence data were collected in a quartz cuvette with $135 \mathrm{~mL}$ of sample. Bimane fluorescence was measured by excitation at $370 \mathrm{~nm}$ with excitation and emission bandwidth passes of $3 \mathrm{~nm}$, and emission spectra were recorded from 400 to $550 \mathrm{~nm}$ in $2 \mathrm{~nm}$ increments with $0.1 \mathrm{~s}$ integration time. Care was taken to extensively rinse and argon-dry the cuvette between individual measurements. To remove background fluorescence, buffer spectra were collected using the same settings, and subtracted from each sample spectrum.

FRET measurements of AF488-AT647N-labeled $\beta$ arr1 constructs were performed as described for bimane measurements, with the following differences: samples were excited at $476 \mathrm{~nm}$ with 3 $\mathrm{nm}$ excitation and $4 \mathrm{~nm}$ emission slit widths. Spectra were collected from $485 \mathrm{~nm}$ to $750 \mathrm{~nm}$ in 1 $\mathrm{nm}$ increments with $0.1 \mathrm{~s}$ integration time. FRET measurements in the absence of NTSR1 were performed in buffer containing $20 \mathrm{mM}$ HEPES pH 7.4, $100 \mathrm{mM} \mathrm{NaCl}$ and $0.004 \%$ LMNG (w/v)/0.0004\% CHS (no NTS). FRET measurements with NTSR1 were done with $0.5 \mu \mathrm{M}$ NTSR1 and $0.5 \mu \mathrm{M}$ diC8-PI(4,5)P2.

1019

1020

1021

1022

1023

1024

1025

1026

1027

1028

1029 NBD and BIM spectra measured on the Tecan Infinite M1000 PRO were collected using 96-well flat black Greiner plates with $100 \mu \mathrm{L}$ of sample at a final concentration of $0.5 \mu \mathrm{M} \beta$ arr1 in buffer containing $20 \mathrm{mM}$ HEPES pH 7.4, $100 \mathrm{mM} \mathrm{NaCl}$ and 0.004\% LMNG (w/v)/0.0004\% CHS. For bimane the following instrument settings were used: excitation: $370 \mathrm{~nm}$, emission 420-500 nm (1 $\mathrm{nm}$ steps) with $20 \mathrm{~s}$ read time and $400 \mathrm{~Hz}$ flash mode. For NBD the following instrument settings were used: excitation: $490 \mathrm{~nm}$, emission $510-580 \mathrm{~nm}$ (1 nm steps) with 20 s read time and 400 $\mathrm{Hz}$ flash mode. Gain and z-position were optimized prior to reading.

Efret values for FRET experiments were calculated as Efret $=\frac{A}{(D+A)}$ and normalized to donor intensity within a given experiment. Scaled FRET values (apo $=100, \min ($ FRET) $=0$ ) were fit to a single exponential decay function $Y=(Y 0-$ plateau $) * e^{-K * x}+$ plateau using the nls function in $\mathrm{R}$ for $\mathrm{EC}_{50}$ values. L167W-293NBD, if fit, was fit using the same function. L68BIM data was fit to a total and non-specific binding model (based on GraphPad Prism 9) to obtain $B_{\max }$ and $K_{d}$ values.

1032

1033

1034

1035

1036

1037

1038

1039

1040

1041

1042

1043

1044

\section{Supplemental Information titles and legends}

Figure S1. Arrestin phosphoinositide binding is required for plasma membrane recruitment to some GPCRs. A) cAMP response in HEK293 cells devoid of $\beta$-arrestins upon stimulation of endogenous $\beta 2 A R$ with $100 \mathrm{nM}$ isoproterenol (iso). Clone 1 (CL1) and Clone 2 (CL2) are independent $\beta$ arr1/2 knock-out cell lines (O'Hare et al. 2017). Data are normalized to response with Forskolin (Fsk)/3-isobutyl-1-methylxanthine (IBMX) and show mean with $95 \%$ confidence intervals ( $n=3$ independent experiments). Two-way analysis of variance (ANOVA), Tukey's multiple comparison test. For CL2 * denotes $p<0.05$ for WT vs. mApple over the interval of 1732 minutes, while $3 Q$ vs. mApple was not significant. For $C L 1{ }^{*}$ denotes $p<0.05$ for WT vs. mApple over the interval of 19-29 minutes, while $3 Q$ vs. mApple was not significant. B)

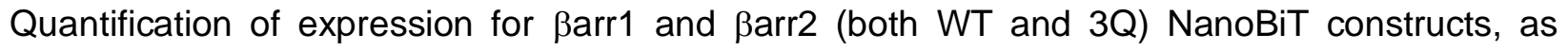
1045 determined by western blot (Supplementary data figure 2). Mean values of 3-4 independent 
1046

1047

1048

1049

1050

1051

1052

1053

1054

1055

1056

1057

1058

1059

1060

1061

1062

1063

1064

1065

1066

1067

1068

1069

1070

1071

1072

1073

1074

1075

1076

1077

1078

1079

1080

1081

1082

1083

1084

1085

1086

1087

1088

1089

1090

1091

1092

1093

1094

1095

experiments were compared by a two-tailed unpaired t-test, where ns denotes $p>0.05$, ${ }^{*} \mathrm{P} \leq$ 0.05. Boxplots: center line, median; box range, 25-75th percentiles; whiskers denote minimummaximum values. Individual points are shown. C) LOF is only weakly correlated with recruitment of WT $\beta$-arrestins. Data are mean LOF and mean WT $\beta$ arr1/2 recruitment. $\beta a r r 1$ recruitment is shown as circles and $\beta$ arr2 recruitment is shown as triangles. Data are colored based on assigned cluster. Dashed line shows expected linear relationship and $R$ is the Pearson coefficient, with 0.51 reflecting a weak negative correlation. D) Plot of LOF data for plasma membrane bystander (CAAX) vs. LOF for direct recruitment. Barr1 recruitment is shown as circles and Barr2 recruitment is shown as triangles. Data are colored based on assigned cluster. Dashed line shows expected linear relationship and $R$ is the Pearson coefficient, with 0.88 reflecting a very strong positive correlation. E) NanoBiT assay for measuring endosome translocation of Barr1. Cartoon of endosome bystander assay (left). Barr1 endosome recruitment data (right) with dashed ellipses to indicate clusters based on CAAX data. $\beta$-arrestin endosome recruitment determined by span of luminescence fold change. Data are mean \pm SEM ( $n=3$ independent experiments). Dashed line indicates three times the maximum signal measured in mock (receptor) transfected cells.

Figure S2. Loss of PIP binding slows $\beta$-arrestin recruitment to cluster 2 GPCRs. A) initial rate (05 minutes post-agonist stimulation) expressed as luminescence fold-change (FC)/min. Data from $\mathrm{n}=3$ independent experiments fit independently (see methods). Boxplots: center line, median; box range, 25-75th percentiles; whiskers denote minimum-maximum values. For each receptor, and for each ßarr1 and Barr2 WT and 3Q were compared by a two-tailed unpaired t-test, where ns denotes $p>0.05,{ }^{*} P \leq 0.05,{ }^{* *} P \leq 0.01,{ }^{* * *} P \leq 0.0001$. B) Data from A) expressed as a difference in rate shows that with the exception of Barr1-TACR1 all cluster 2 receptors show faster recruitment of WT $\beta$-arrestin1/2 than corresponding $3 Q$ mutant. Data are mean \pm SEM $(n=3$ independent experiments).

Figure S3. Arrestin recruitment to NTSR1 mutants can be compared by NanoBiT assay. A) Expression of NTSR1 constructs in HEK293A cells used for NanoBiT assays. Boxplots: center line, median; box range, 25-75th percentiles; whiskers denote minimum-maximum values. Individual points are shown. Values are mean, relative to NTSR1-WT ( $\mathrm{n}=4$ independent experiments). For each construct, a comparison to NTSR1-WT by a two-tailed unpaired Wilcoxon test was performed, where ns denotes $p>0.05,{ }^{*} \mathrm{P} \leq 0.05$. B) NanoBiT Emax for Sm-ßarr1 interaction with Lg-CAAX for cells expressing NTSR1-WT as a function of mean fluorescence intensity (MFI), as determined by cell-surface staining. Amount of NTSR1-WT DNA transfected is written; arrow denotes $200 \mathrm{ng}$, the amount used in recruitment assays in Figure 2. C) As B, except the pEC50 of recruitment response upon NTS stimulation is plotted instead of Emax. Emax and pEC50 are mean \pm SD ( $n=2$ independent experiments), MFI determined by cell-surface staining are mean \pm SD ( $n=2$ independent experiments).

Figure S4. PIP binding stabilizes core-engaged arrestin complexes. A) LOF in complexing efficiency as determined by SEC. LOF $=1$ corresponds to complete loss of complex formation, while LOF $=0$ corresponds to no difference in complexing efficiency between WT and 3Q ßarr1 ( $n=5$ independent experiments). Boxplots: center line, median; box range, 25-75th percentiles; whiskers denote minimum-maximum values. Individual points are shown. compared by a twotailed unpaired t-test, where ${ }^{* * *} P \leq 0.0001$. B) Structure of transition from inactive (PDB: 1G4M) to active (PDB: 4JQI) Barr1 involves displacement of the ßarr1 C-tail (dark grey) by V2Rpp (blue). Two cysteine residues were added to a cys-less ßarr1 background at positions A12 and V387 (pink spheres). These positions were labeled with fluorophores that, through FRET, allow for monitoring the position of the C-tail. C) When labeled with a FRET pair, ßarr1-12C/387C shows a high-FRET state in the absence of V2Rpp, and a low-FRET state when the ßarr1 C-tail is 
displaced by V2Rpp (left). FRET measured when $\beta$ arr1 (WT or 3Q)-12C/387C-AF488-AT647N is bound to V2Rpp $(0.5 \mu \mathrm{M})$, NTSR1 (GRK5p, $0.5 \mu \mathrm{M})$. All samples containing NTSR1 were

1098 supplemented with diC8-PI(4,5)P2 $(0.5 \mu \mathrm{M})$. Apo $\beta$ arr1 (WT or 3Q)-12C/387C-AF488-AT647N was normalized to 1.0 and $\beta$ arr1 (WT or 3Q)-12C/387C-AF488-AT647N + V2Rpp (10 $\mu \mathrm{M})$ was normalized 0.0 for each experiment ( $n=3$ independent measurements) (right). Boxplots: center line, median; box range, 25-75th percentiles; whiskers denote minimum-maximum values. 1102 Individual points are shown.

1103

1104

Figure S5. PIP2 allosterically triggers movement of the arrestin C-tail, but not release. A-B) L68Cbimane responses. \%apo is scaled such that the fluorescence intensity (at $\lambda$ max) for apo arrestin is $100 \%$ and each condition is scaled as a factor of apo. ND denotes not determined values. Values for $\mathrm{B}_{\max }$ (max response) and $\mathrm{K}_{d}$ (based on single-site binding fitting) are provided and ranges in parentheses correspond to $95 \% \mathrm{Cl}$. Points are mean and error bars reflect $95 \% \mathrm{Cl}(\mathrm{n}=3$ independent experiments). C-D) L167W-293C-NBD responses. \%apo is scaled such that the fluorescence intensity (at $\lambda \max$ ) for apo arrestin is $100 \%$ and each condition is scaled as a factor of apo. Points represent values ( $\mathrm{n}=1$ independent experiments). E-F) A12C-V387C-AF488AT647N responses. \%FRET is scaled such that apo arrestin is $100 \%$ and the highest concentration of V2Rpp (100 uM) is $0 \%$. INF denotes infinite upper bound. ND denotes not determined values. Range of EC50 values is indicated in parentheses and represents $95 \% \mathrm{Cl}$. Points represent mean and error bars reflect $95 \% \mathrm{Cl}$ ( $\mathrm{n}=3$ independent experiments). 


\section{References}

1118

1119

1120

1121

1122

1123

1124

1125

1126

1127

1128

1129

1130

1131

1132

1133

1134

1135

1136

1137

1138

1139

1140

1141

1142

1143

1144

1145

1146

1147

1148

1149

1150

1151

1152

1153

1154

1155
Baidya, M., Kumari, P., Dwivedi-Agnihotri, H., Pandey, S., Chaturvedi, M., Stepniewski, T.M., Kawakami, K., Cao, Y., Laporte, S.A., Selent, J., et al. (2020a). Key phosphorylation sites in GPCRs orchestrate the contribution of beta-Arrestin 1 in ERK1/2 activation. EMBO Rep 21, e49886.

Baidya, M., Kumari, P., Dwivedi-Agnihotri, H., Pandey, S., Sokrat, B., Sposini, S., Chaturvedi, M., Srivastava, A., Roy, D., Hanyaloglu, A.C., et al. (2020b). Genetically encoded intrabody sensors report the interaction and trafficking of beta-arrestin 1 upon activation of G-protein-coupled receptors. J Biol Chem 295, 10153-10167.

Beyett, T.S., Fraley, A.E., Labudde, E., Patra, D., Coleman, R.C., Eguchi, A., Glukhova, A., Chen, Q., Williams, R.M., Koch, W.J., et al. (2019). Perturbation of the interactions of calmodulin with GRK5 using a natural product chemical probe. Proc Natl Acad Sci U S A 116, 15895-15900.

Cahill, T.J., 3rd, Thomsen, A.R., Tarrasch, J.T., Plouffe, B., Nguyen, A.H., Yang, F., Huang, L.Y., Kahsai, A.W., Bassoni, D.L., Gavino, B.J., et al. (2017). Distinct conformations of GPCR-betaarrestin complexes mediate desensitization, signaling, and endocytosis. Proc Natl Acad Sci U S A 114, 2562-2567.

Chen, Q., Perry, N.A., Vishnivetskiy, S.A., Berndt, S., Gilbert, N.C., Zhuo, Y., Singh, P.K., Tholen, J., Ohi, M.D., Gurevich, E.V., et al. (2017). Structural basis of arrestin-3 activation and signaling. Nat Commun 8, 1427.

Chen, Q., Zhuo, Y., Sharma, P., Perez, I., Francis, D.J., Chakravarthy, S., Vishnivetskiy, S.A., Berndt, S., Hanson, S.M., Zhan, X., et al. (2021). An Eight Amino Acid Segment Controls Oligomerization and Preferred Conformation of the two Non-visual Arrestins. J Mol Biol 433, 166790.

De Matteis, M.A., and Godi, A. (2004). Pl-loting membrane traffic. Nat Cell Biol 6, 487-492.

DeFea, K.A., Zalevsky, J., Thoma, M.S., Dery, O., Mullins, R.D., and Bunnett, N.W. (2000). betaarrestin-dependent endocytosis of proteinase-activated receptor 2 is required for intracellular targeting of activated ERK1/2. J Cell Biol 148, 1267-1281.

Dery, O., Thoma, M.S., Wong, H., Grady, E.F., and Bunnett, N.W. (1999). Trafficking of proteinase-activated receptor-2 and beta-arrestin-1 tagged with green fluorescent protein. betaArrestin-dependent endocytosis of a proteinase receptor. J Biol Chem 274, 18524-18535.

Di Paolo, G., and De Camilli, P. (2006). Phosphoinositides in cell regulation and membrane dynamics. Nature 443, 651-657.

Dixon, A.S., Schwinn, M.K., Hall, M.P., Zimmerman, K., Otto, P., Lubben, T.H., Butler, B.L., Binkowski, B.F., Machleidt, T., Kirkland, T.A., et al. (2016). NanoLuc Complementation Reporter Optimized for Accurate Measurement of Protein Interactions in Cells. ACS Chem Biol 11, 400408.

Eichel, K., Jullie, D., Barsi-Rhyne, B., Latorraca, N.R., Masureel, M., Sibarita, J.B., Dror, R.O., and von Zastrow, M. (2018). Catalytic activation of beta-arrestin by GPCRs. Nature 557, 381-386. 
1156 Eichel, K., Jullie, D., and von Zastrow, M. (2016). beta-Arrestin drives MAP kinase signalling from clathrin-coated structures after GPCR dissociation. Nat Cell Biol 18, 303-310.

1158 Feinstein, T.N., Wehbi, V.L., Ardura, J.A., Wheeler, D.S., Ferrandon, S., Gardella, T.J., and Vilardaga, J.P. (2011). Retromer terminates the generation of cAMP by internalized PTH receptors. Nat Chem Biol 7, 278-284.

1161 Feinstein, T.N., Yui, N., Webber, M.J., Wehbi, V.L., Stevenson, H.P., King, J.D., Jr., Hallows, K.R., Brown, D., Bouley, R., and Vilardaga, J.P. (2013). Noncanonical control of vasopressin receptor type 2 signaling by retromer and arrestin. J Biol Chem 288, 27849-27860.

1164 Ferrandon, S., Feinstein, T.N., Castro, M., Wang, B., Bouley, R., Potts, J.T., Gardella, T.J., and Vilardaga, J.P. (2009). Sustained cyclic AMP production by parathyroid hormone receptor endocytosis. Nat Chem Biol 5, 734-742.

1167 Gaidarov, I., Krupnick, J.G., Falck, J.R., Benovic, J.L., and Keen, J.H. (1999). Arrestin function in $\mathrm{G}$ protein-coupled receptor endocytosis requires phosphoinositide binding. EMBO J 18, 871-881.

Ghosh, E., Dwivedi, H., Baidya, M., Srivastava, A., Kumari, P., Stepniewski, T., Kim, H.R., Lee, M.H., van Gastel, J., Chaturvedi, M., et al. (2019). Conformational Sensors and Domain Swapping Reveal Structural and Functional Differences between beta-Arrestin Isoforms. Cell Rep 28, 32873299 e3286.

1173 Grundmann, M., Merten, N., Malfacini, D., Inoue, A., Preis, P., Simon, K., Ruttiger, N., Ziegler, N., Benkel, T., Schmitt, N.K., et al. (2018). Lack of beta-arrestin signaling in the absence of active G proteins. Nat Commun 9, 341.

1176 Hanyaloglu, A.C., and von Zastrow, M. (2008). Regulation of GPCRs by endocytic membrane trafficking and its potential implications. Annu Rev Pharmacol Toxicol 48, 537-568. K.C., Glenn, J.S., Skiniotis, G., et al. (2020). Structure of the neurotensin receptor 1 in complex with beta-arrestin 1. Nature 579, 303-308.

1181 Innamorati, G., Sadeghi, H., and Birnbaumer, M. (1998a). Transient phosphorylation of the V1a vasopressin receptor. J Biol Chem 273, 7155-7161. recycling of the V2 vasopressin receptor. Proc Natl Acad Sci U S A 95, 2222-2226. Ishida, S., Arang, N., et al. (2019). Illuminating G-Protein-Coupling Selectivity of GPCRs. Cell 177, 1933-1947 e1925.

1188 Irannejad, R., Tomshine, J.C., Tomshine, J.R., Chevalier, M., Mahoney, J.P., Steyaert, J., 1189 Rasmussen, S.G., Sunahara, R.K., El-Samad, H., Huang, B., et al. (2013). Conformational 

(2017). Regulation of clathrin-mediated endocytosis by hierarchical allosteric activation of AP2. J Cell Biol 216, 167-179. regulation of endosomal GPCR/beta-arrestin complexes and trafficking by MAPK. J Biol Chem 289, 23302-23317. arrestin. Proc Natl Acad Sci U S A 109, 18407-18412.

1202 Kim, Y.J., Hofmann, K.P., Ernst, O.P., Scheerer, P., Choe, H.W., and Sommer, M.E. (2013).

1204 Komolov, K.E., and Benovic, J.L. (2018). G protein-coupled receptor kinases: Past, present and future. Cell Signal 41, 17-24. dephosphorylation by vesicular acidification. J Biol Chem 272, 5-8.

1209 Kumari, P., Srivastava, A., Banerjee, R., Ghosh, E., Gupta, P., Ranjan, R., Chen, X., Gupta, B., arrestin complex. Nat Commun 7, 13416.

1212 Latorraca, N.R., Masureel, M., Hollingsworth, S.A., Heydenreich, F.M., Suomivuori, C.M., Brinton, Phosphorylation Patterns Orchestrate Arrestin-Mediated Signaling. Cell 183, 1813-1825 e1818.

Lee, Y., Warne, T., Nehme, R., Pandey, S., Dwivedi-Agnihotri, H., Chaturvedi, M., Edwards, P.C., Garcia-Nafria, J., Leslie, A.G.W., Shukla, A.K., et al. (2020). Molecular basis of beta-arrestin coupling to formoterol-bound beta1-adrenoceptor. Nature $583,862-866$.

1218 Lobingier, B.T., and von Zastrow, M. (2019). When trafficking and signaling mix: How subcellular location shapes $\mathrm{G}$ protein-coupled receptor activation of heterotrimeric G proteins. Traffic 20, 130136.

1221 Luttrell, L.M., Wang, J., Plouffe, B., Smith, J.S., Yamani, L., Kaur, S., Jean-Charles, P.Y., 1222 Gauthier, C., Lee, M.H., Pani, B., et al. (2018). Manifold roles of beta-arrestins in GPCR signaling elucidated with siRNA and CRISPR/Cas9. Sci Signal 11. S1P1 receptor phosphorylation, internalization, and interaction with Rab proteins: effects of sphingosine 1-phosphate, FTY720-P, phorbol esters, and paroxetine. Biosci Rep 38.

1227 Milano, S.K., Kim, Y.M., Stefano, F.P., Benovic, J.L., and Brenner, C. (2006). Nonvisual arrestin 
Nakagawa, T., and Asahi, M. (2013). beta1-adrenergic receptor recycles via a membranous organelle, recycling endosome, by binding with sorting nexin27. J Membr Biol 246, 571-579.

1232 Nguyen, A.H., Thomsen, A.R.B., Cahill, T.J., 3rd, Huang, R., Huang, L.Y., Marcink, T., Clarke, O.B., Heissel, S., Masoudi, A., Ben-Hail, D., et al. (2019). Structure of an endosomal signaling GPCR-G protein-beta-arrestin megacomplex. Nat Struct Mol Biol 26, 1123-1131. Messer, K., Sunahara, R., et al. (2017). Genetic evidence that beta-arrestins are dispensable for the initiation of beta2-adrenergic receptor signaling to ERK. Sci Signal 10. arrestin with $G$ protein-coupled receptors during clathrin-mediated endocytosis dictates the profile of receptor resensitization. J Biol Chem 274, 32248-32257. determinants underlying the formation of stable intracellular $G$ protein-coupled receptor-betaarrestin complexes after receptor endocytosis*. J Biol Chem 276, 19452-19460. major classes of receptors. J Biol Chem 275, 17201-17210. of arrestins, G proteins and Src kinase. Traffic 19, 58-82.

1260 Shukla, A.K., Manglik, A., Kruse, A.C., Xiao, K., Reis, R.I., Tseng, W.C., Staus, D.P., Hilger, D.,

Rajagopal, S., and Shenoy, S.K. (2018). GPCR desensitization: Acute and prolonged phases. Cell Signal 41, 9-16.

Ranjan, R., Dwivedi, H., Baidya, M., Kumar, M., and Shukla, A.K. (2017). Novel Structural Insights into GPCR-beta-Arrestin Interaction and Signaling. Trends Cell Biol 27, 851-862.

Reiter, E., Ahn, S., Shukla, A.K., and Lefkowitz, R.J. (2012). Molecular mechanism of betaarrestin-biased agonism at seven-transmembrane receptors. Annu Rev Pharmacol Toxicol 52, 179-197.

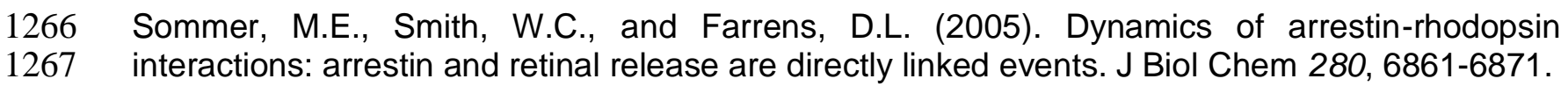

Sente, A., Peer, R., Srivastava, A., Baidya, M., Lesk, A.M., Balaji, S., Shukla, A.K., Babu, M.M., and Flock, T. (2018). Molecular mechanism of modulating arrestin conformation by GPCR phosphorylation. Nat Struct Mol Biol 25, 538-545. Uysal, S., Huang, L.Y., et al. (2013). Structure of active beta-arrestin-1 bound to a G-proteincoupled receptor phosphopeptide. Nature 497, 137-141.

Shukla, A.K., Westfield, G.H., Xiao, K., Reis, R.I., Huang, L.Y., Tripathi-Shukla, P., Qian, J., Li, S., Blanc, A., Oleskie, A.N., et al. (2014). Visualization of arrestin recruitment by a G-proteincoupled receptor. Nature 512, 218-222. 
Sommer, M.E., Smith, W.C., and Farrens, D.L. (2006). Dynamics of arrestin-rhodopsin interactions: acidic phospholipids enable binding of arrestin to purified rhodopsin in detergent. J Biol Chem 281, 9407-9417.

1271 Staus, D.P., Hu, H., Robertson, M.J., Kleinhenz, A.L.W., Wingler, L.M., Capel, W.D., Latorraca, N.R., Lefkowitz, R.J., and Skiniotis, G. (2020). Structure of the M2 muscarinic receptor-betaarrestin complex in a lipid nanodisc. Nature 579, 297-302.

1274 Sun, Y., Carroll, S., Kaksonen, M., Toshima, J.Y., and Drubin, D.G. (2007). Ptdlns(4,5)P2 turnover is required for multiple stages during clathrin- and actin-dependent endocytic internalization. J Cell Biol 177, 355-367.

1277 Tewson, P.H., Martinka, S., Shaner, N.C., Hughes, T.E., and Quinn, A.M. (2016). New DAG and cAMP Sensors Optimized for Live-Cell Assays in Automated Laboratories. J Biomol Screen 21, 298-305.

Thomsen, A.R.B., Plouffe, B., Cahill, T.J., 3rd, Shukla, A.K., Tarrasch, J.T., Dosey, A.M., Kahsai, A.W., Strachan, R.T., Pani, B., Mahoney, J.P., et al. (2016). GPCR-G Protein-beta-Arrestin Super-Complex Mediates Sustained G Protein Signaling. Cell 166, 907-919.

1283 Toth, D.J., Toth, J.T., Gulyas, G., Balla, A., Balla, T., Hunyady, L., and Varnai, P. (2012). Acute depletion of plasma membrane phosphatidylinositol 4,5-bisphosphate impairs specific steps in endocytosis of the G-protein-coupled receptor. J Cell Sci 125, 2185-2197.

1286 Trapaidze, N., Gomes, I., Bansinath, M., and Devi, L.A. (2000). Recycling and resensitization of delta opioid receptors. DNA Cell Biol 19, 195-204.

1288 Yin, W., Li, Z., Jin, M., Yin, Y.L., de Waal, P.W., Pal, K., Yin, Y., Gao, X., He, Y., Gao, J., et al. (2019). A complex structure of arrestin-2 bound to a $G$ protein-coupled receptor. Cell Res 29, $1290 \quad 971-983$.

1291 Zhuang, T., Vishnivetskiy, S.A., Gurevich, V.V., and Sanders, C.R. (2010). Elucidation of inositol hexaphosphate and heparin interaction sites and conformational changes in arrestin-1 by solution nuclear magnetic resonance. Biochemistry 49, 10473-10485.

1294 Zhuo, Y., Vishnivetskiy, S.A., Zhan, X., Gurevich, V.V., and Klug, C.S. (2014). Identification of 
bioRxiv preprint doi: https://doi.org/10.1101/2021.10.09.463790; this version posted October 10, 2021. The copyright holder for this preprint (which was not certified by peer review) is the author/funder, who has granted bioRxiv a license to display the preprint in perpetuity. It is made

Figure 1 available under aCC-BY-NC-ND 4.0 International license.

A

B

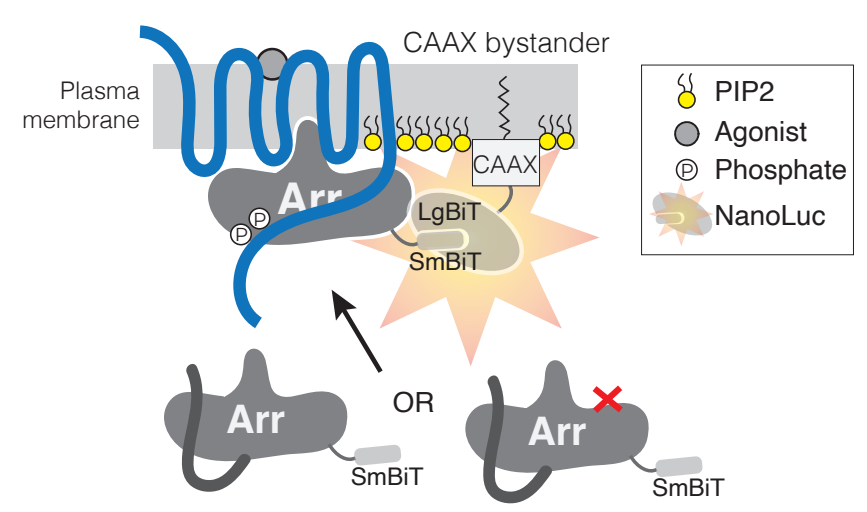

Barr1

WT
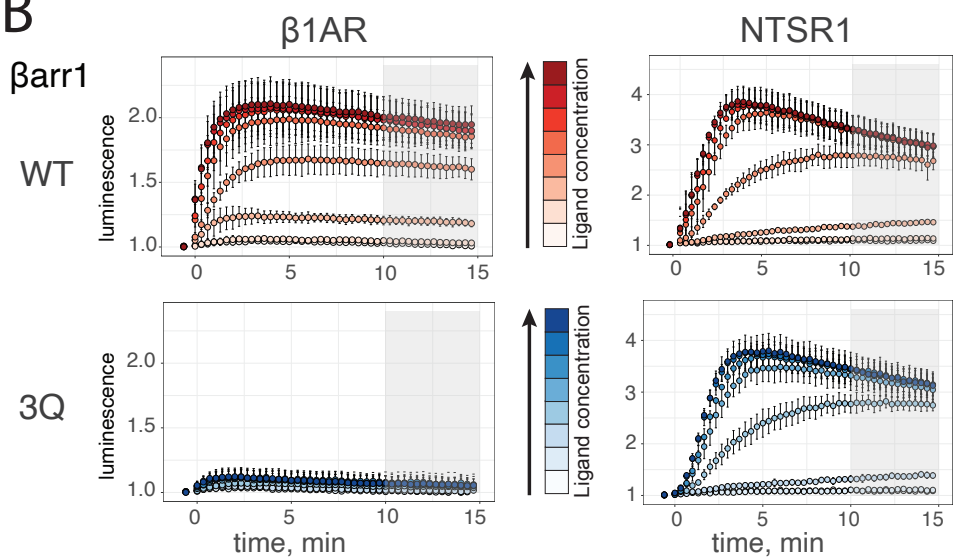

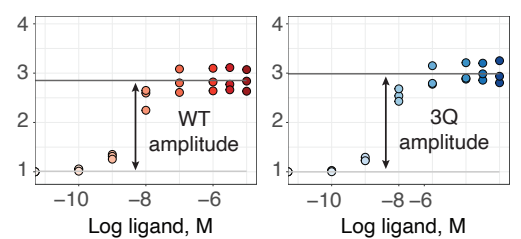

C
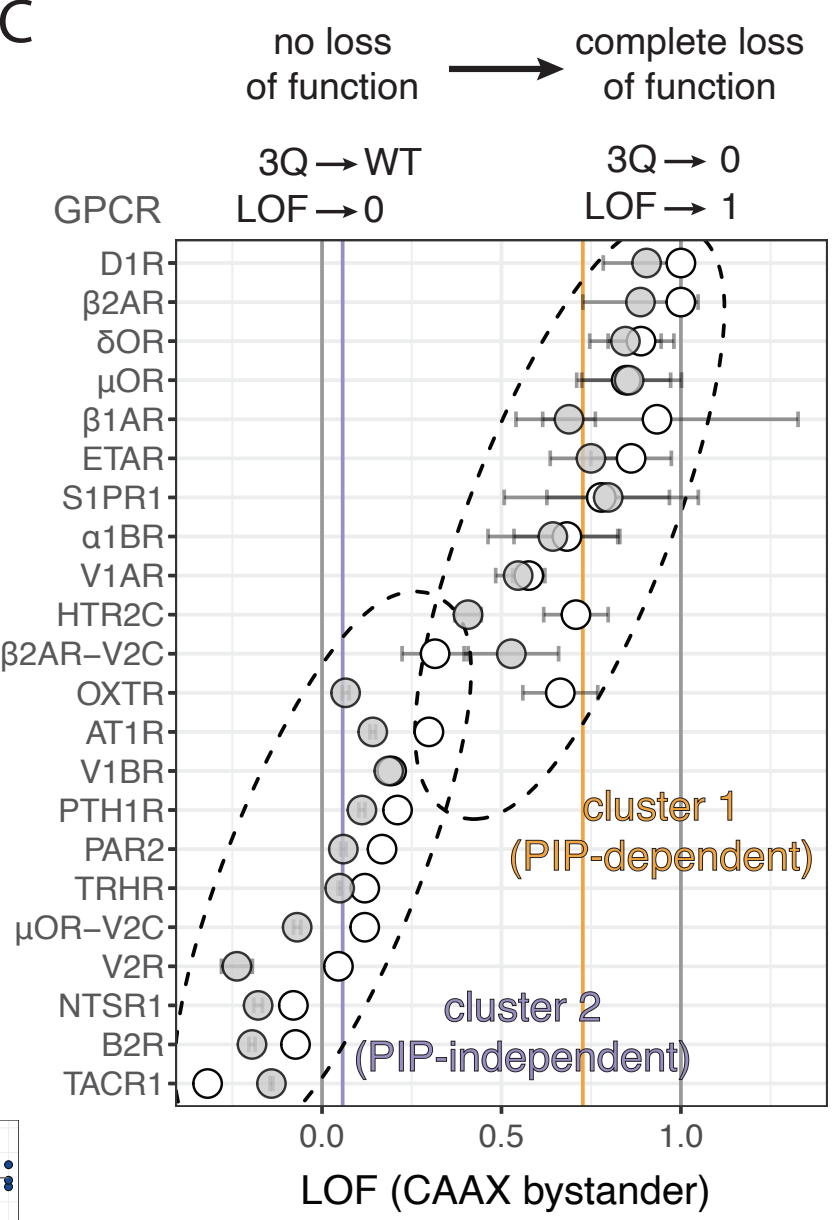

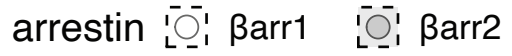


bioRxiv preprint doi: https://doi.org/10.1101/2021.10.09.463790; this version posted October 10, 2021. The copyright holder for this preprint (which was not certified by peer review) is the author/funder, who has granted bioRxiv a license to display the preprint in perpetuity. It is made available under aCC-BY-NC-ND 4.0 International license.

Figure 2

A

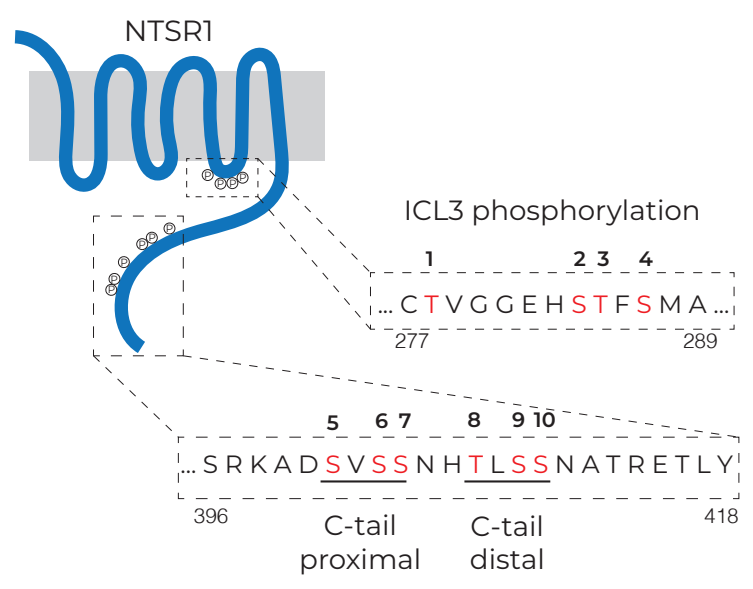

B

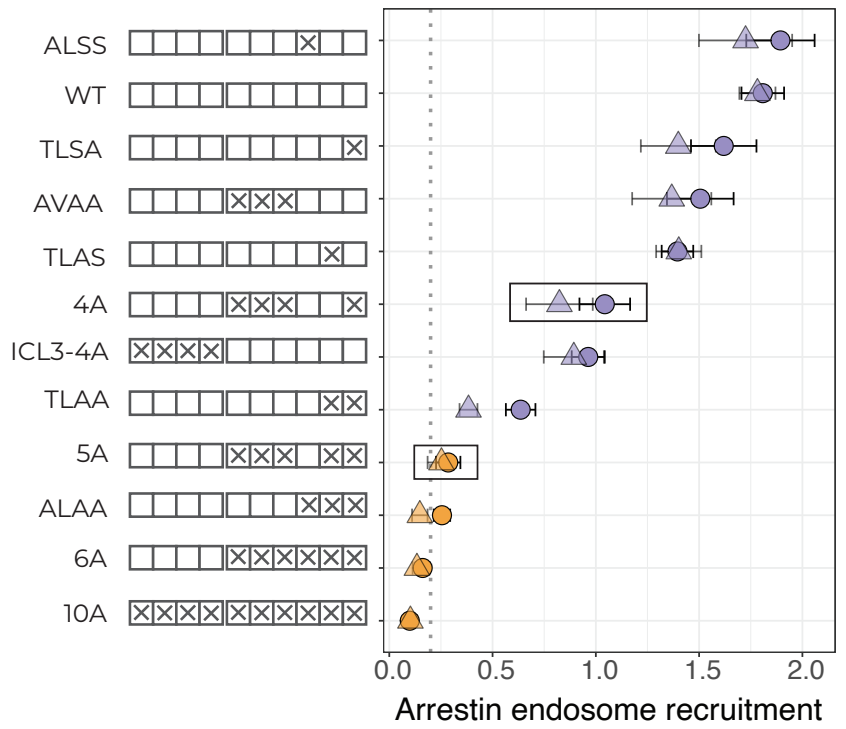

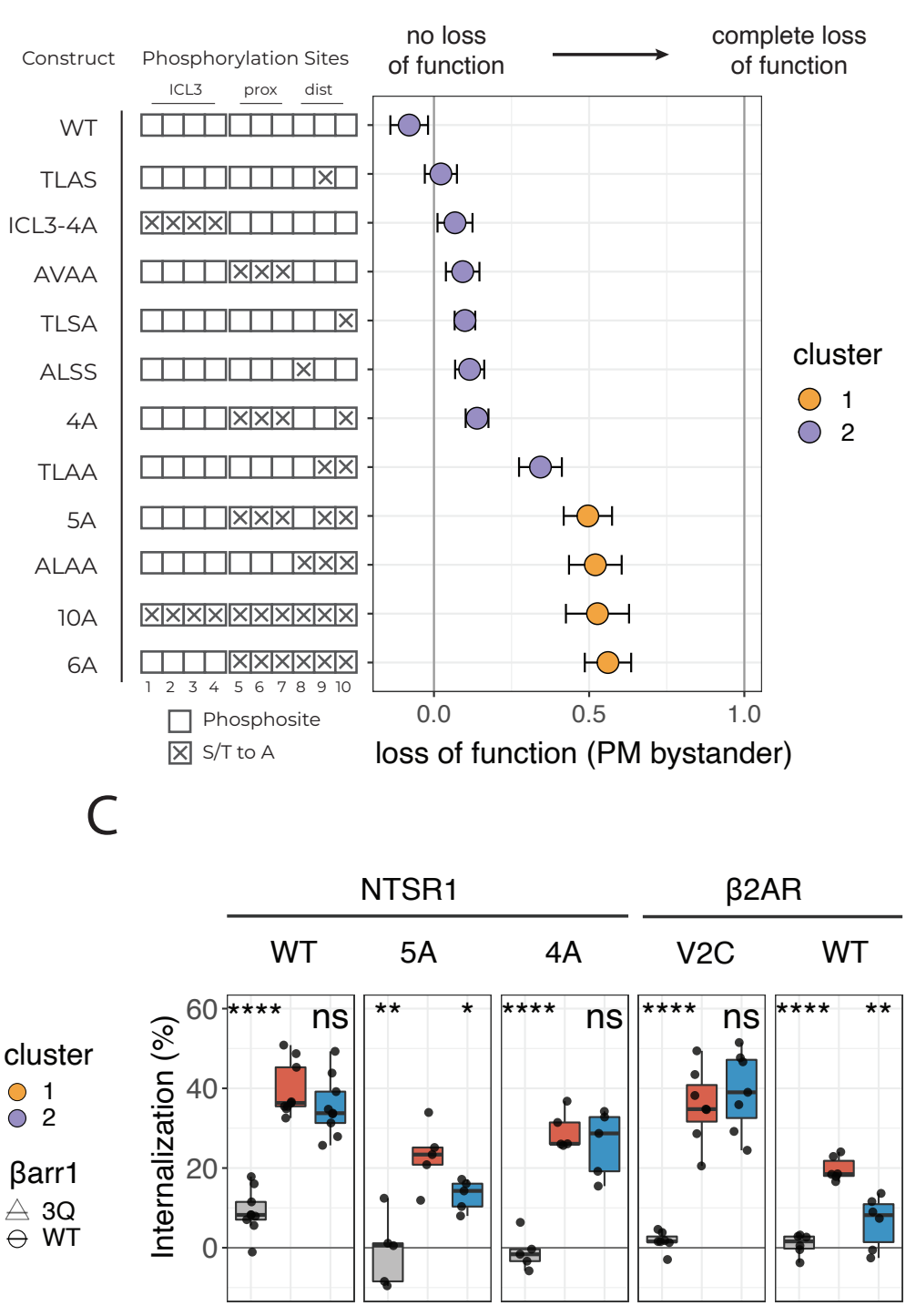

Barr1 审 Mock 豉 WT 审 3Q 
bioRxiv preprint doi: https://doi.org/10.1101/2021.10.09.463790; this version posted October 10, 2021. The copyright holder for this preprint (which was not certified by peer review) is the author/funder, who has granted bioRxiv a license to display the preprint in perpetuity. It is made available under aCC-BY-NC-ND 4.0 International license.

\section{Figure 3}

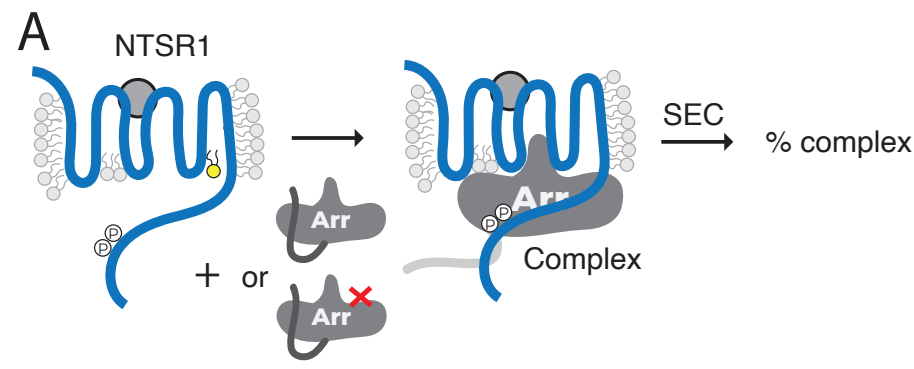

B
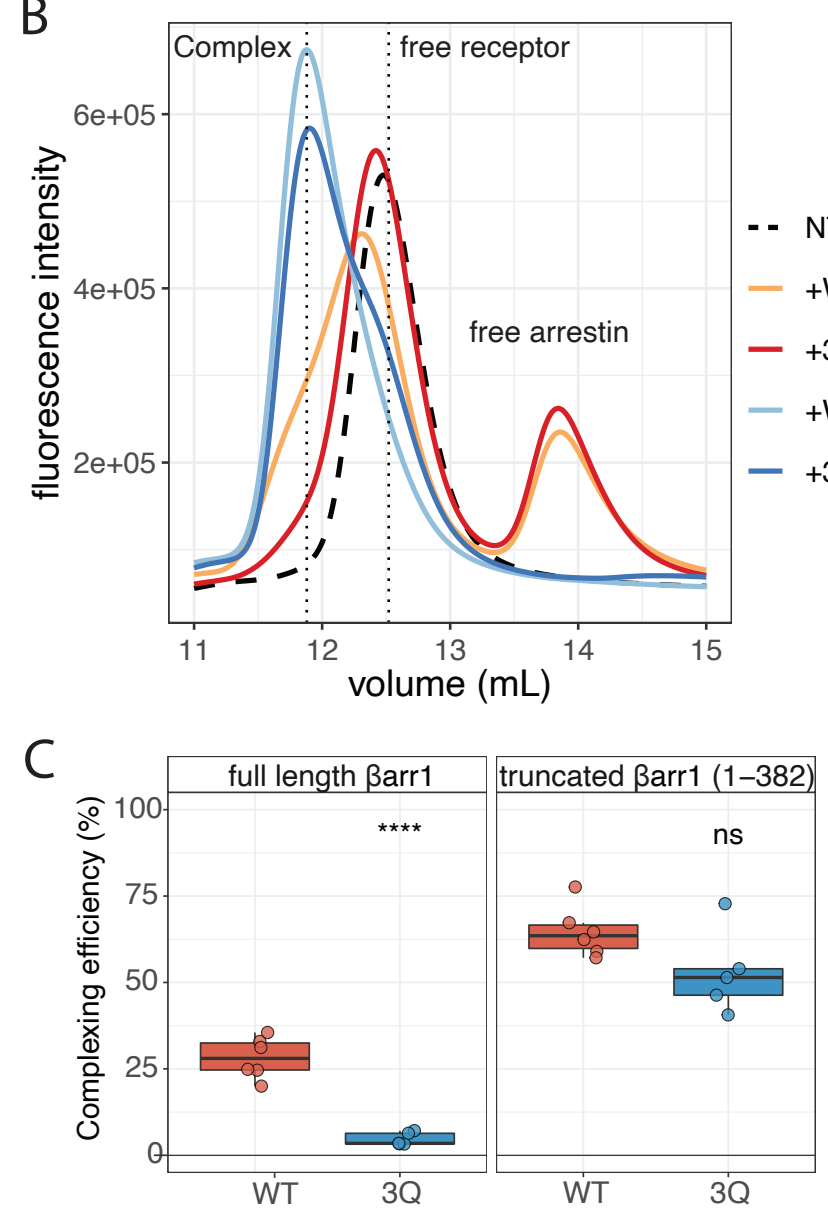

D

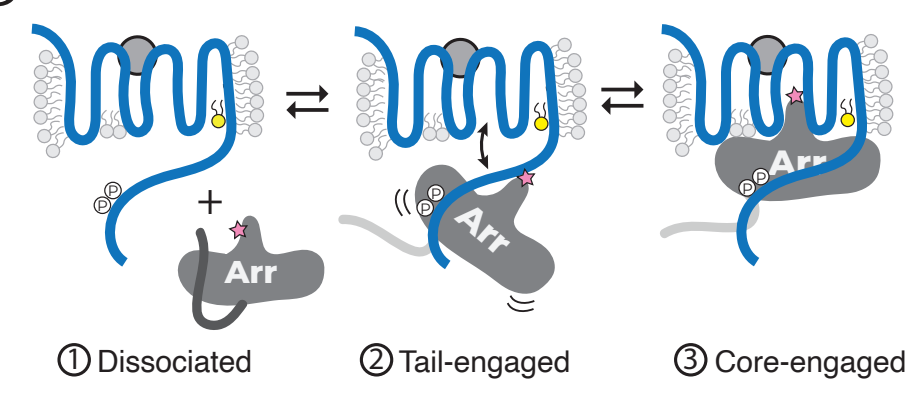

E

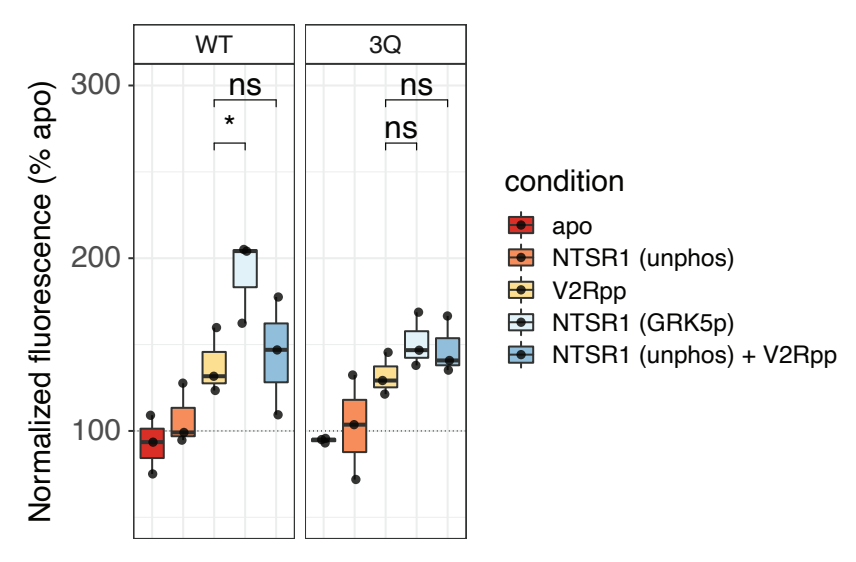

$\mathrm{F}$

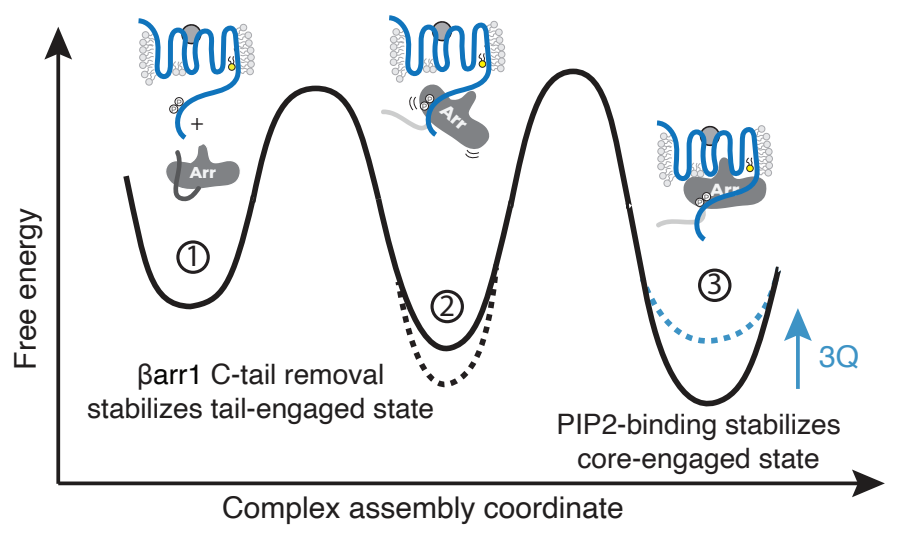


bioRxiv preprint doi: https://doi.org/10.1101/2021.10.09.463790; this version posted October 10, 2021. The copyright holder for this preprint (which was not certified by peer review) is the author/funder, who has granted bioRxiv a license to display the preprint in perpetuity. It is made available under aCC-BY-NC-ND 4.0 International license.

\section{Figure 4}

A

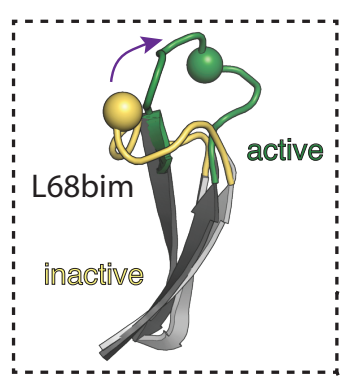

Finger loop

B

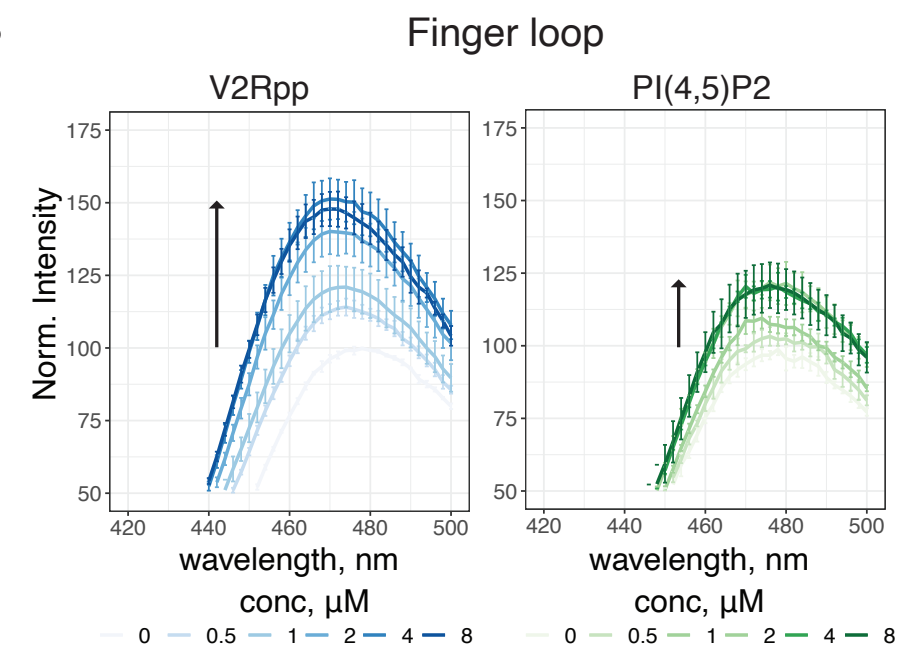

$\mathrm{N}$ lobe

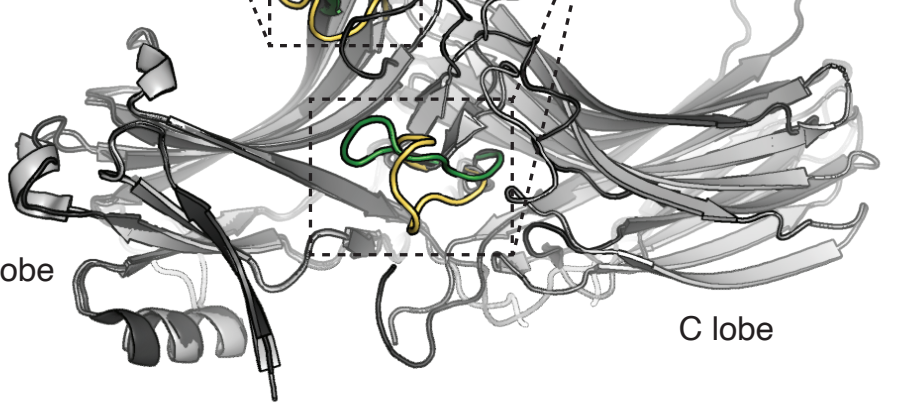

$0-0.5-1-2-4-8$
D

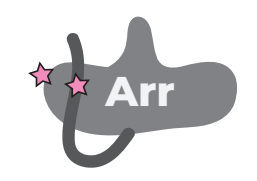

Inactive (high FRET)

"Gate loop<smiles>c1ccc2ccccc2c1</smiles>
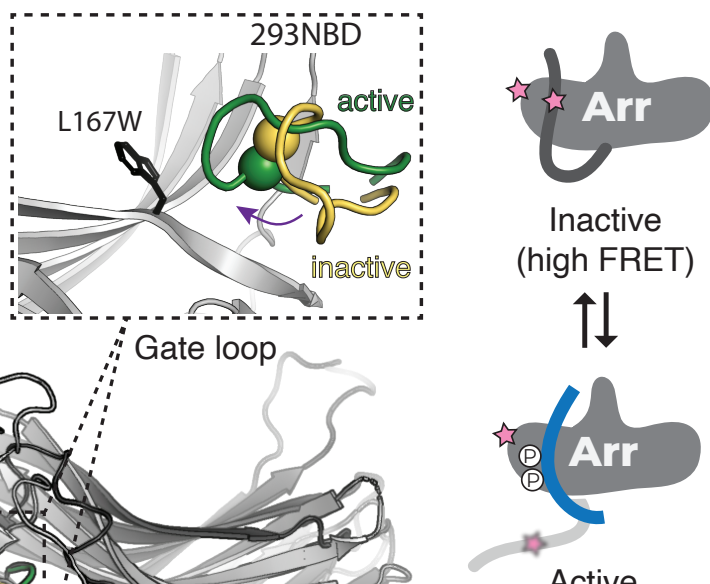

Active (low FRET)

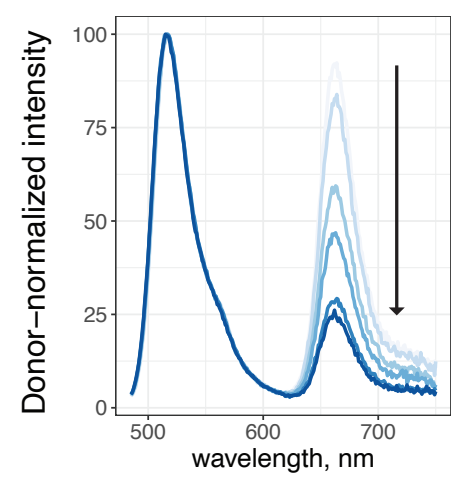

V2Rpp, $\mu \mathrm{M}$

0

-0.1
$-\quad 0.5$

$-1$

$-5$

$-10$

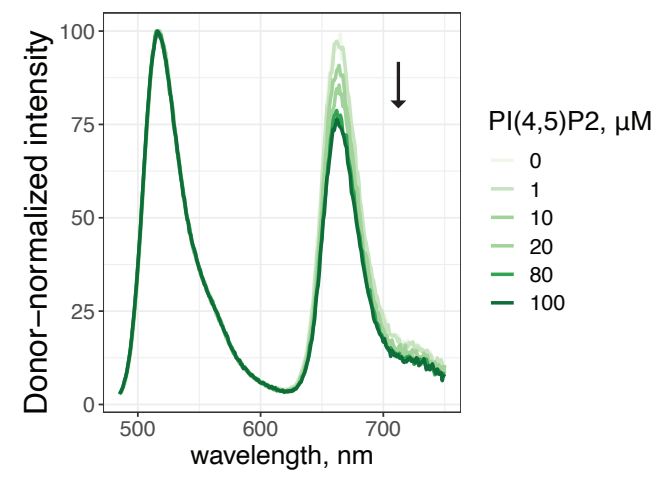

C

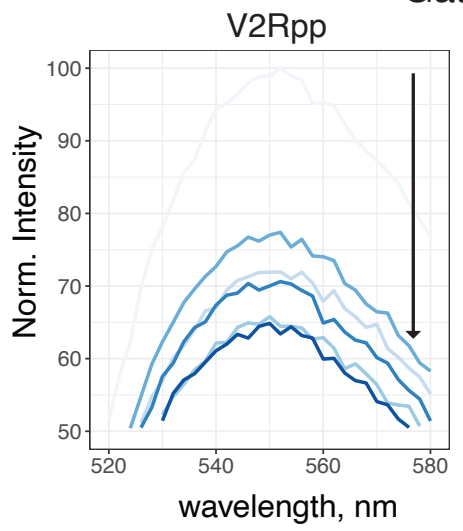

conc, $\mu \mathrm{M}$

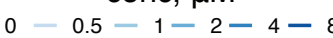

$\mathrm{PI}(4,5) \mathrm{P} 2$

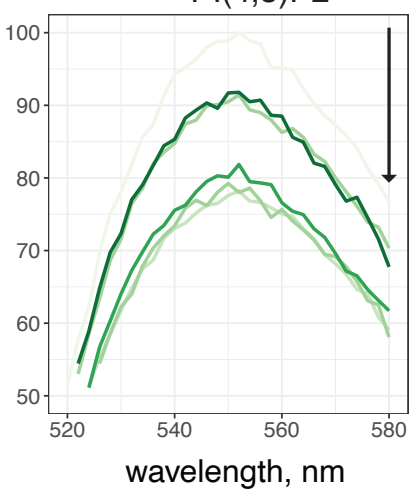

conc, $\mu \mathrm{M}$
$0=0.5-1-2-4-8$

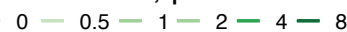


bioRxiv preprint doi: https://doi.org/10.1101/2021.10.09.463790; this version posted October 10, 2021. The copyright holder for this preprint (which was not certified by peer review) is the author/funder, who has granted bioRxiv a license to display the preprint in perpetuity. It is made available under aCC-BY-NC-ND 4.0 International license.

\section{Figure 5}
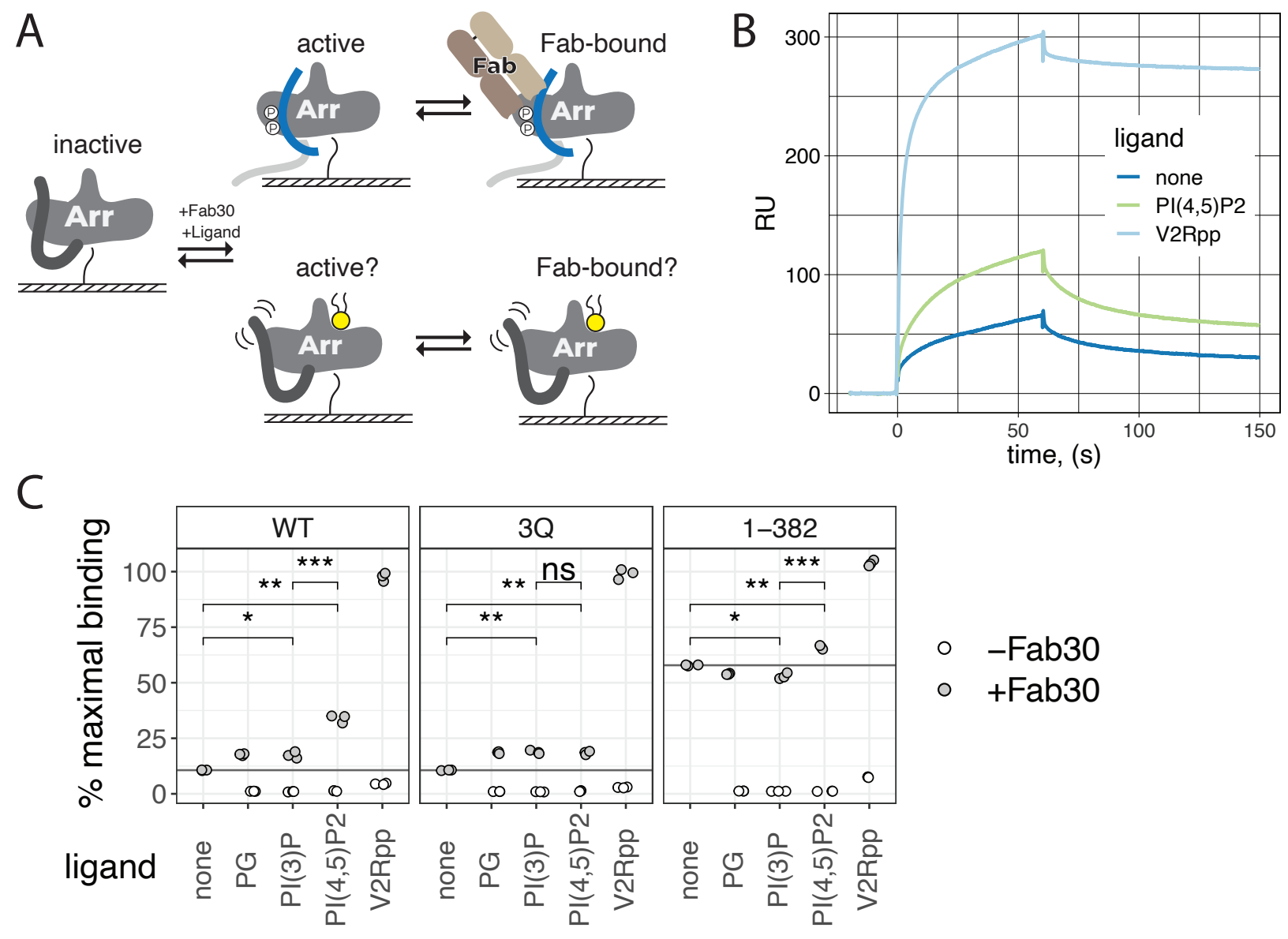


\section{Figure 6}

Class A: PIP-dependent GPCR

Class B: PIP-independent GPCR
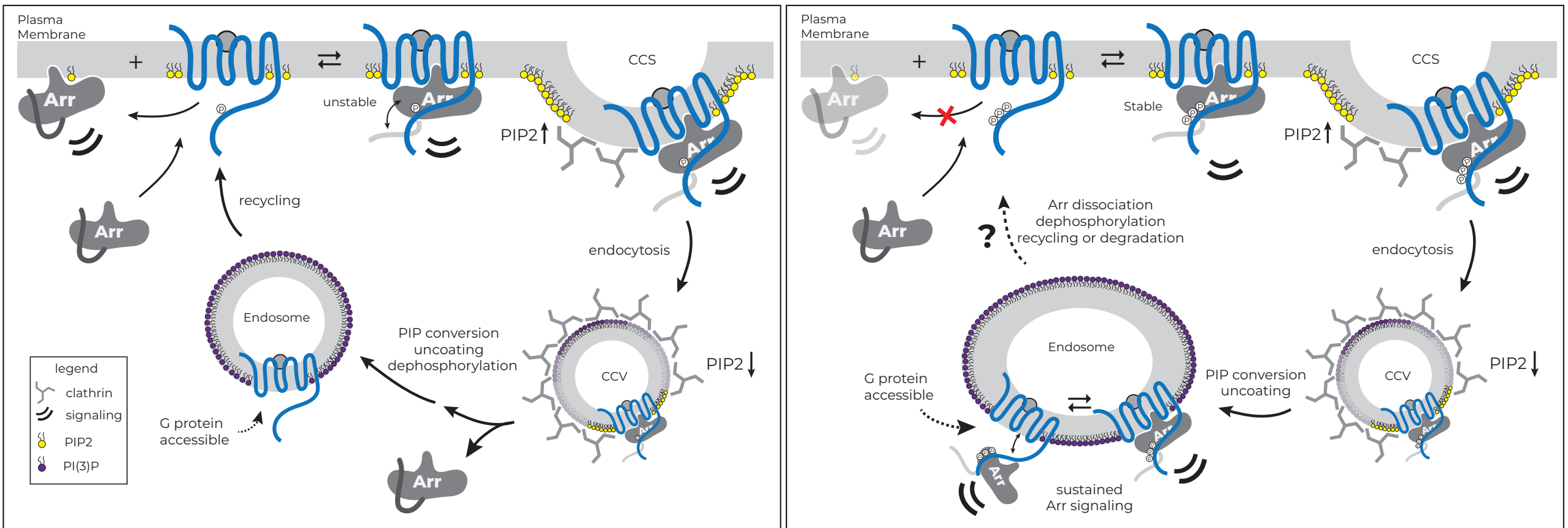
bioRxiv preprint doi: https://doi.org/10.1101/2021.10.09.463790; this version posted October 10, 2021. The copyright holder for this preprint Supryleichmasnat fertifieg bepery reviews is the author/funder, who has granted bioRxiv a license to display the preprint in perpetuity. It is made

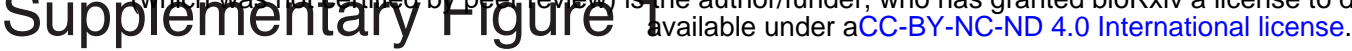

A

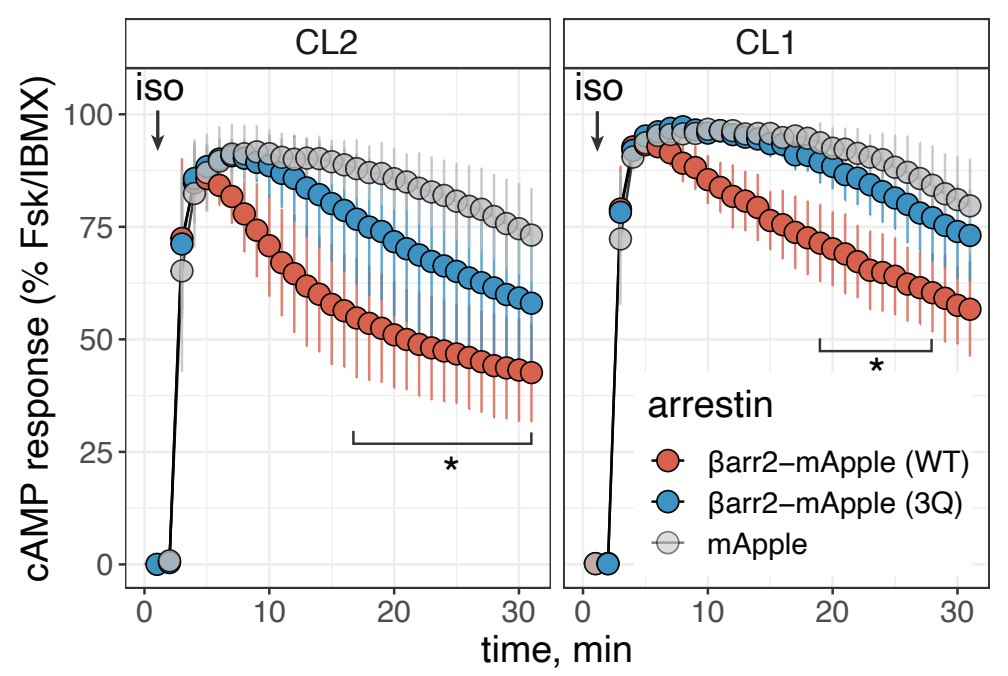

C

$1.0{ }_{31 \mathrm{AR}}^{0}$ DOR

SQR $\triangle$ S1PR1 S1PR1

a1BR HTR2C

Q S OXTR $B 1 A R$

a1BR V1AR

0.5 V1AR

HTR2C B2AR-V2C

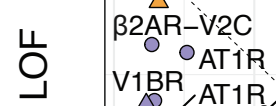

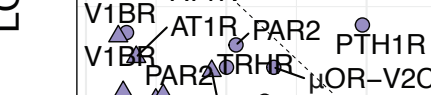

0.0 OXTPTH1R V2R

TRHB $\triangle \mu \mathrm{OR}-\mathrm{V} 2 \mathrm{C}$

NTSRATSRA

TACR1 B2R

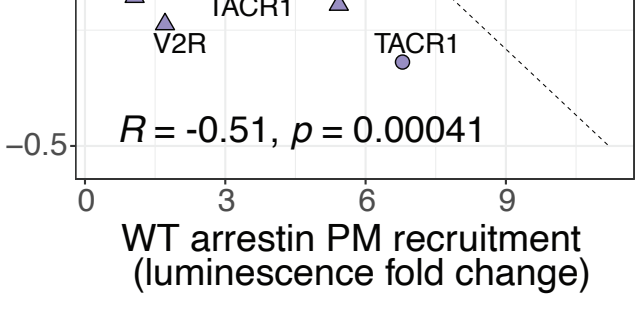

$E$
B
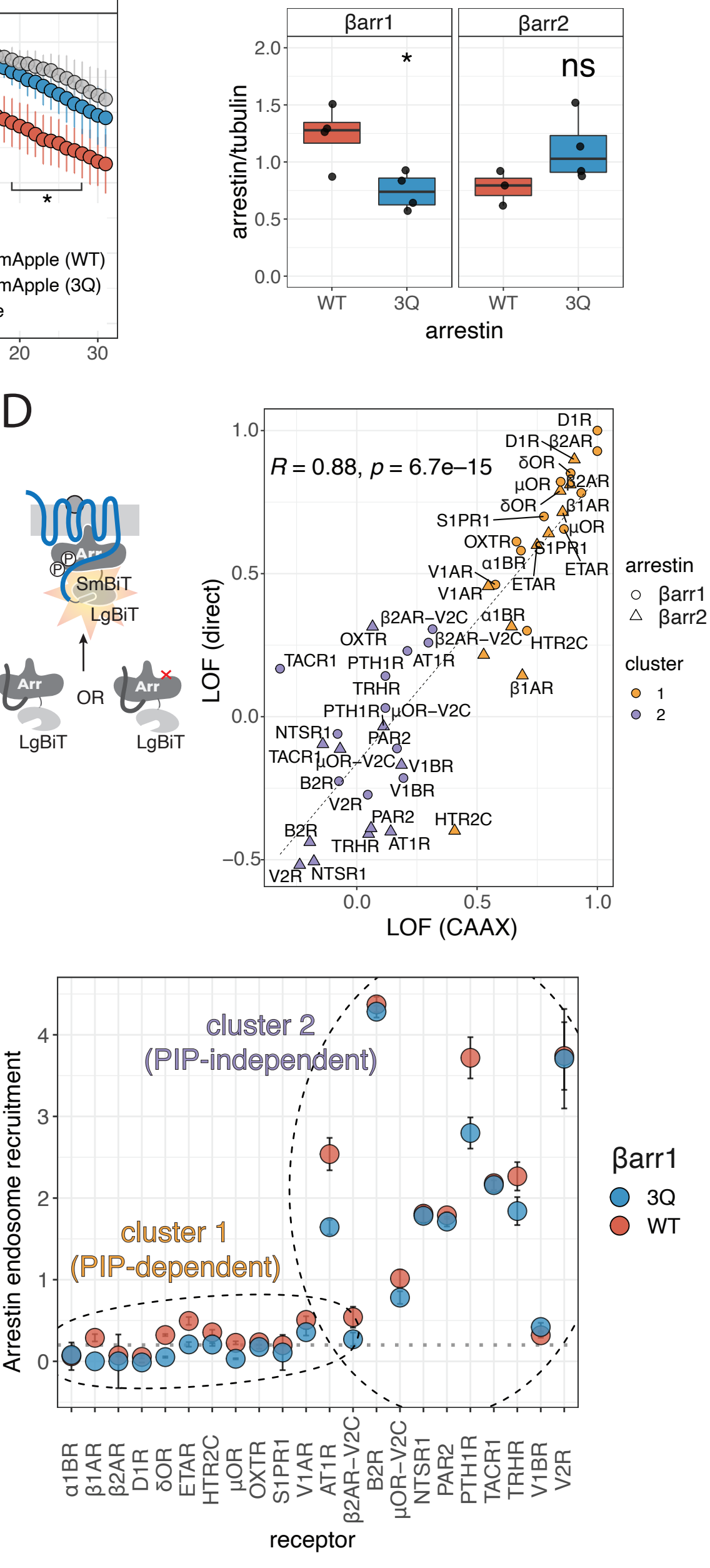
bioRxiv preprint doi: https://doi.org/10.1101/2021.10.09.463790; this version posted October 10, 2021. The copyright holder for this preprint (which was not certified by peer review) is the author/funder, who has granted bioRxiv a license to display the preprint in perpetuity. It is made available under aCC-BY-NC-ND 4.0 International license.

\section{Supplementary Figure 3}

A

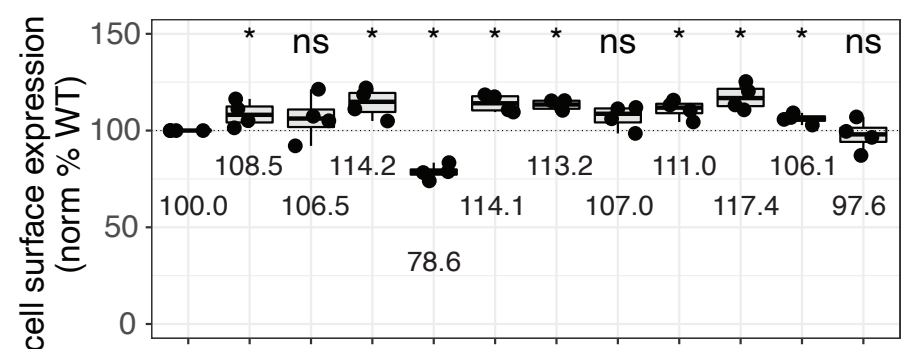

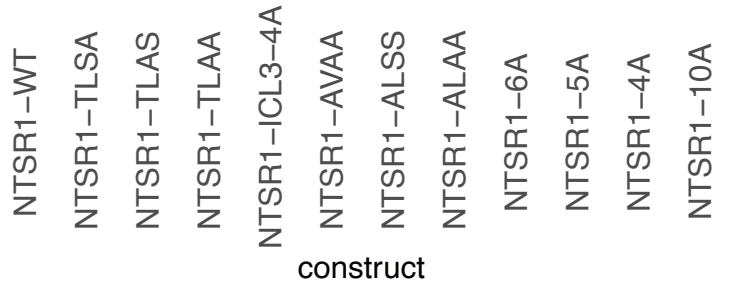

B

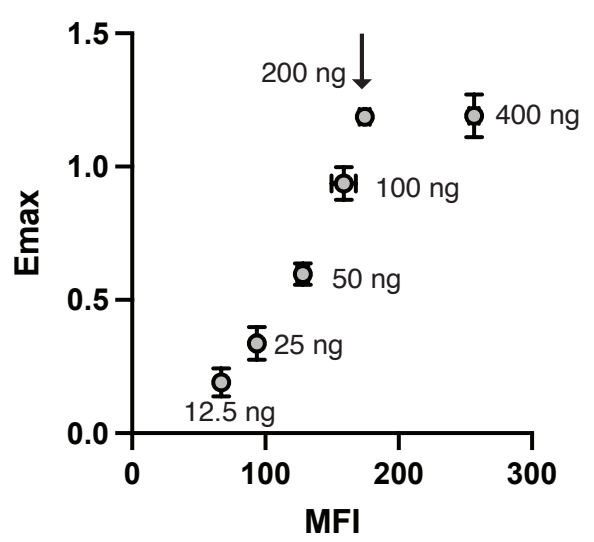

C

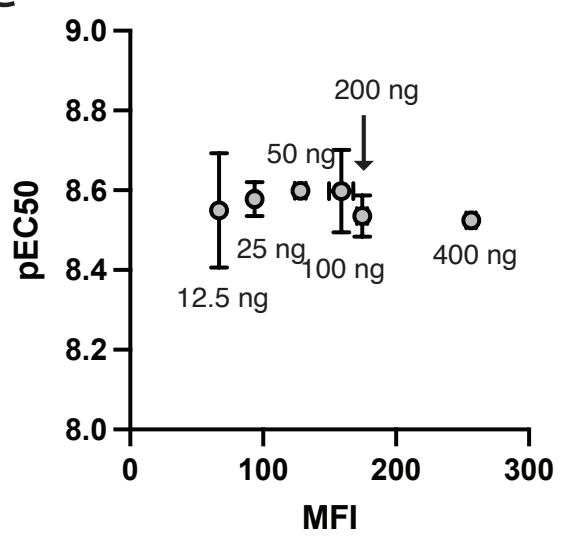


bioRxiv preprint doi: https://doi.org/10.1101/2021.10.09.463790; this version posted October 10, 2021. The copyright holder for this preprint (which was not certified by peer review) is the author/funder, who has granted bioRxiv a license to display the preprint in perpetuity. It is made Supplementary Figure Zavailable under acc-BY-NC-ND 4.0 International license.
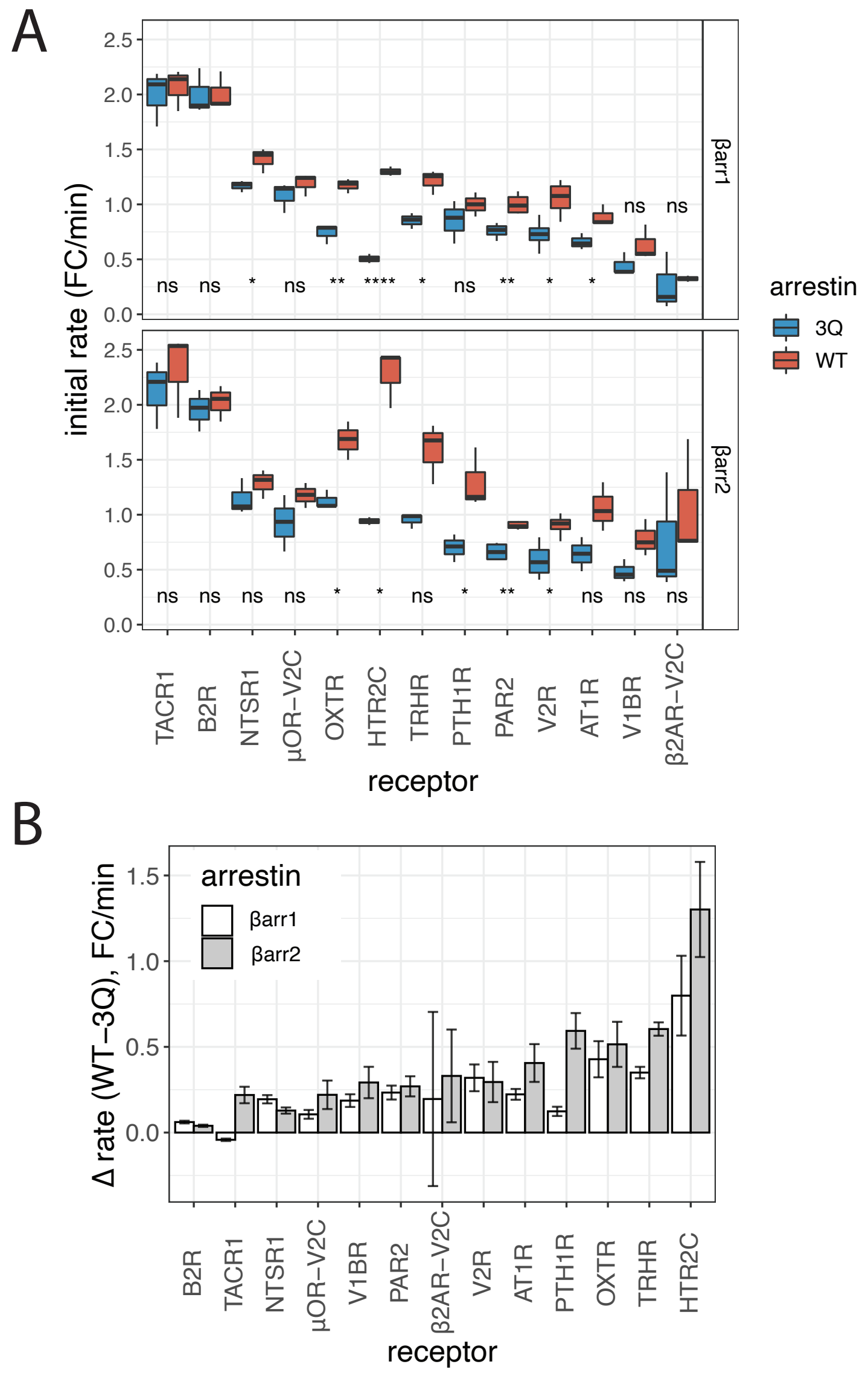
bioRxiv preprint doi: https://doi.org/10.1101/2021.10.09.463790; this version posted October 10, 2021. The copyright holder for this preprint (which was not certified by peer review) is the author/funder, who has granted bioRxiv a license to display the preprint in perpetuity. It is made Supplementary Figure 4 ayailable under aCC-BY-NC-ND 4.0 International license.

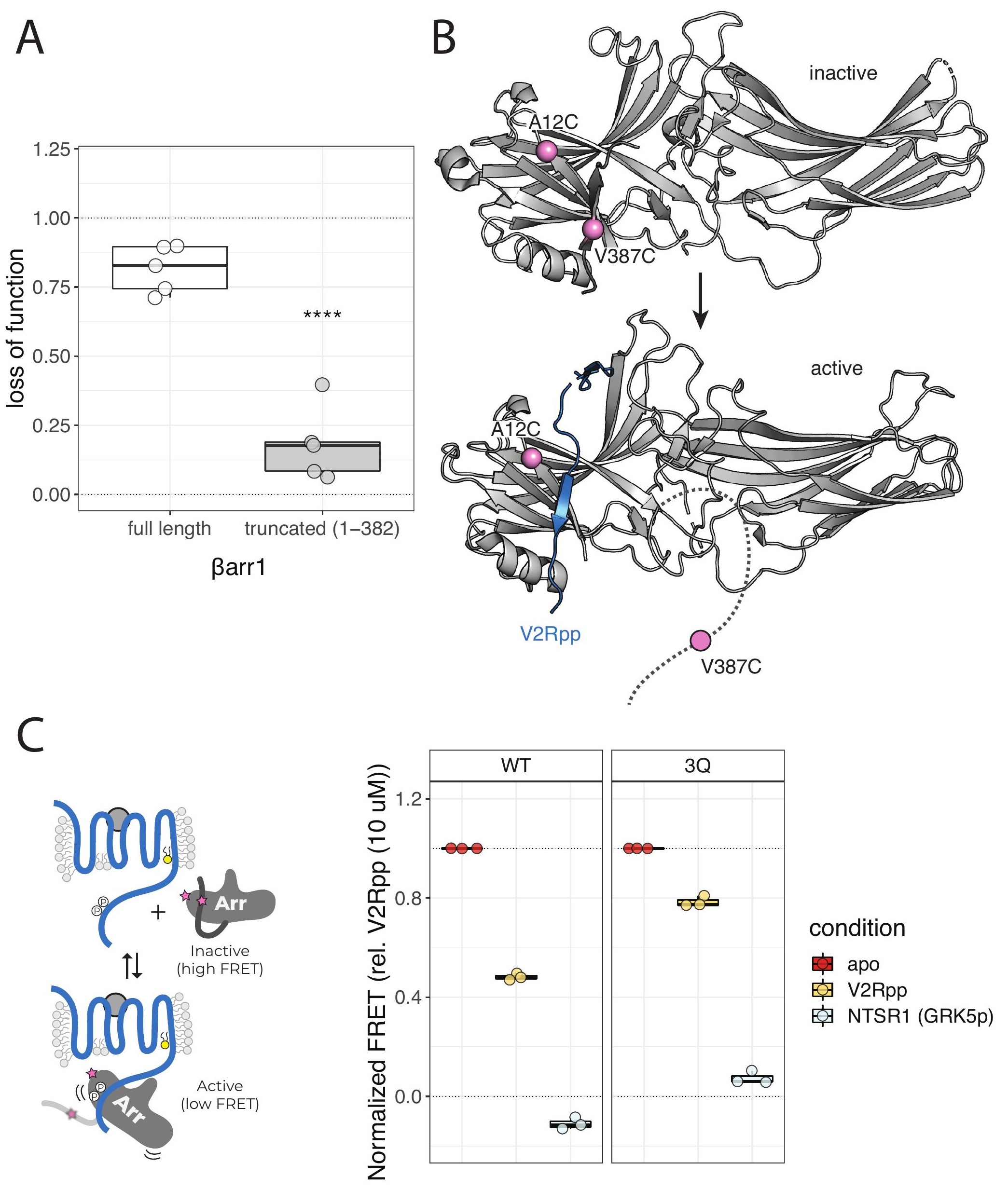


bioRxiv preprint doi: https://doi.org/10.1101/2021.10.09.463790; this version posted October 10, 2021. The copyright holder for this preprint (which was not certified by peer review) is the author/funder, who has granted bioRxiv a license to display the preprint in perpetuity. It is made

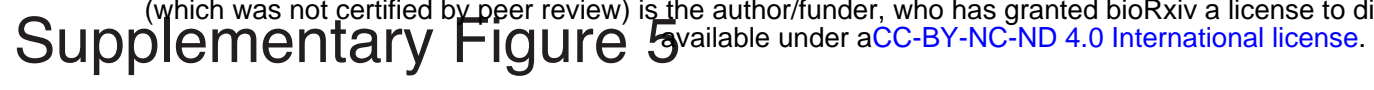
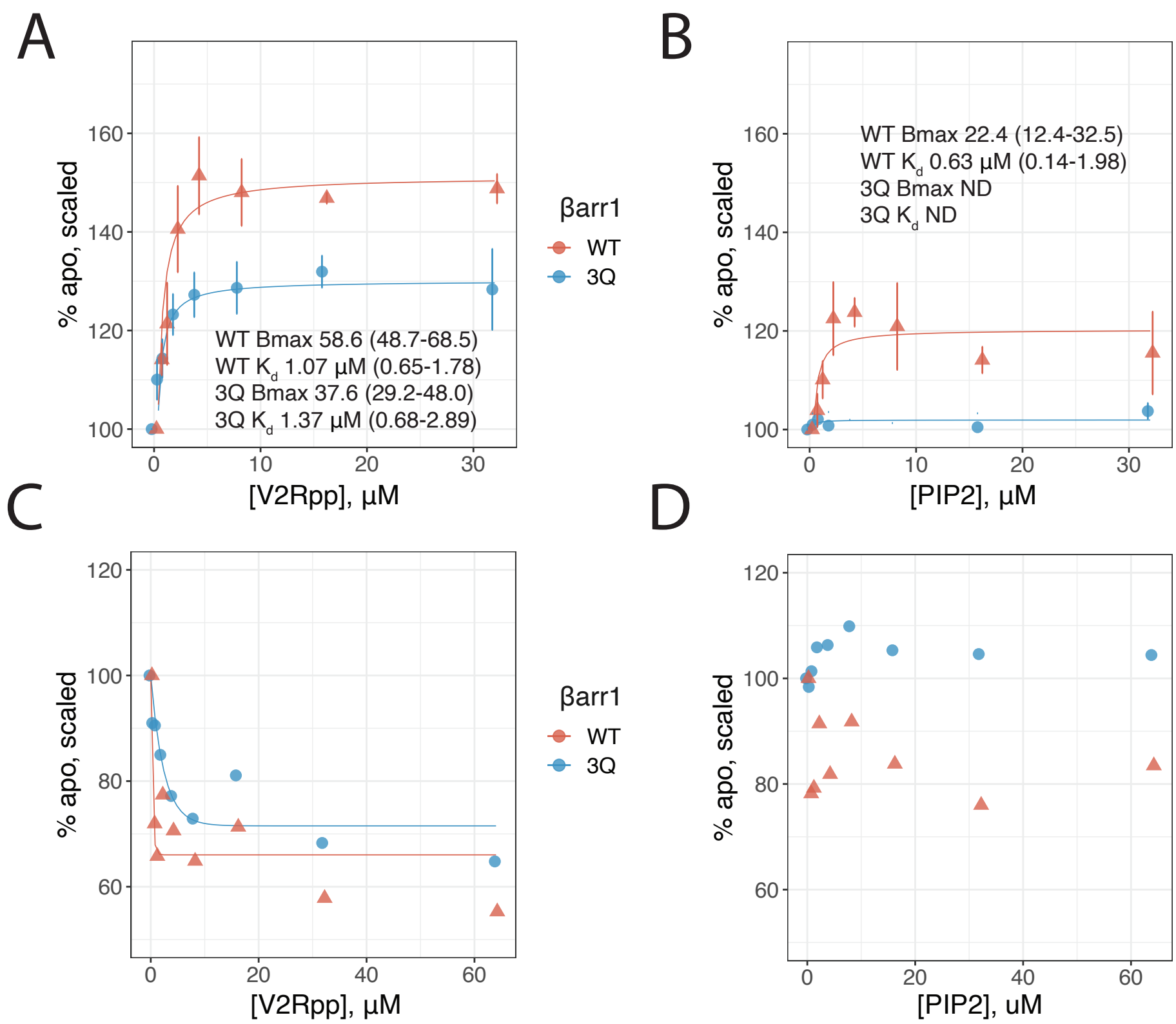

Barr1

- WT

- $3 Q$

E
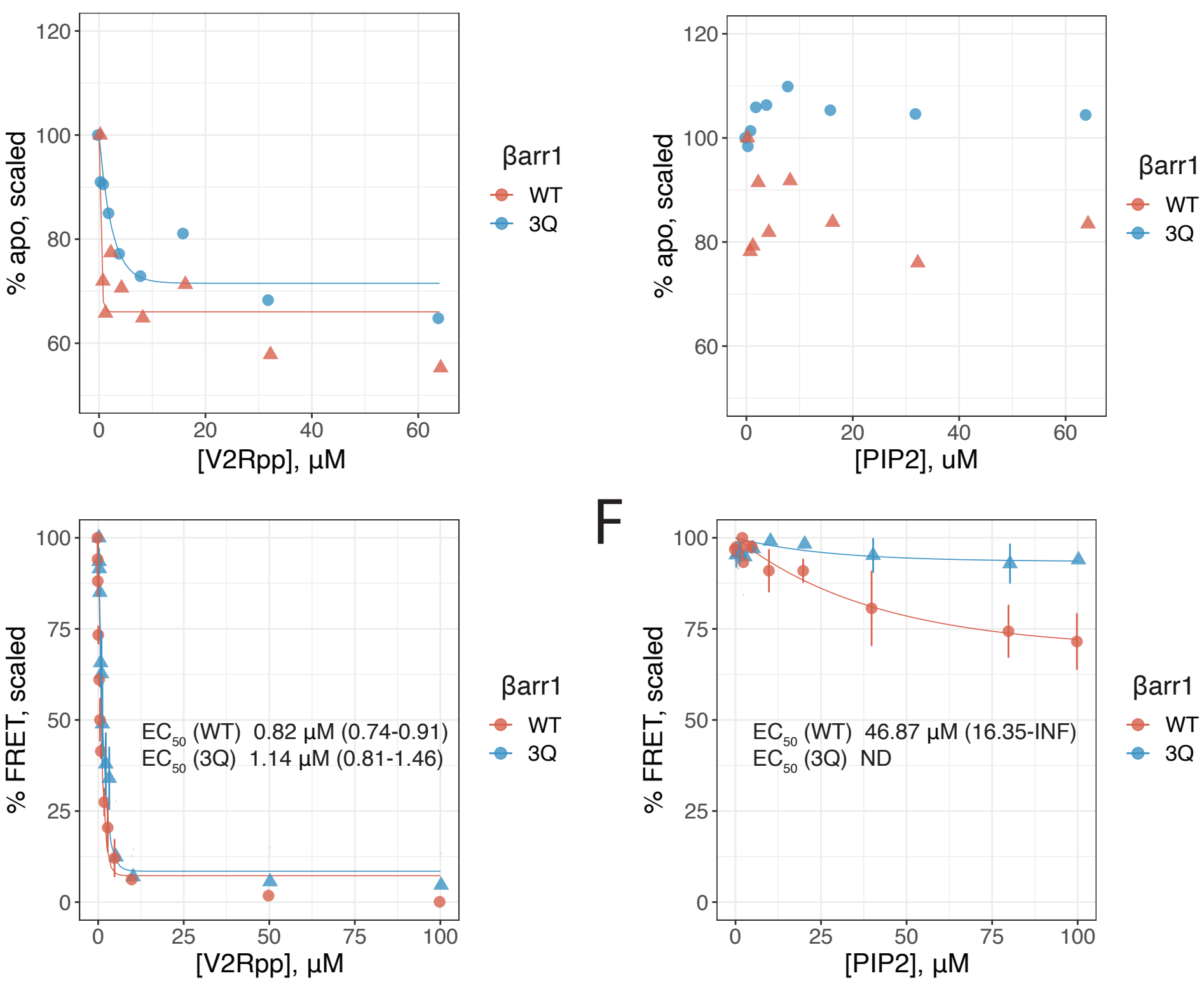

$E$

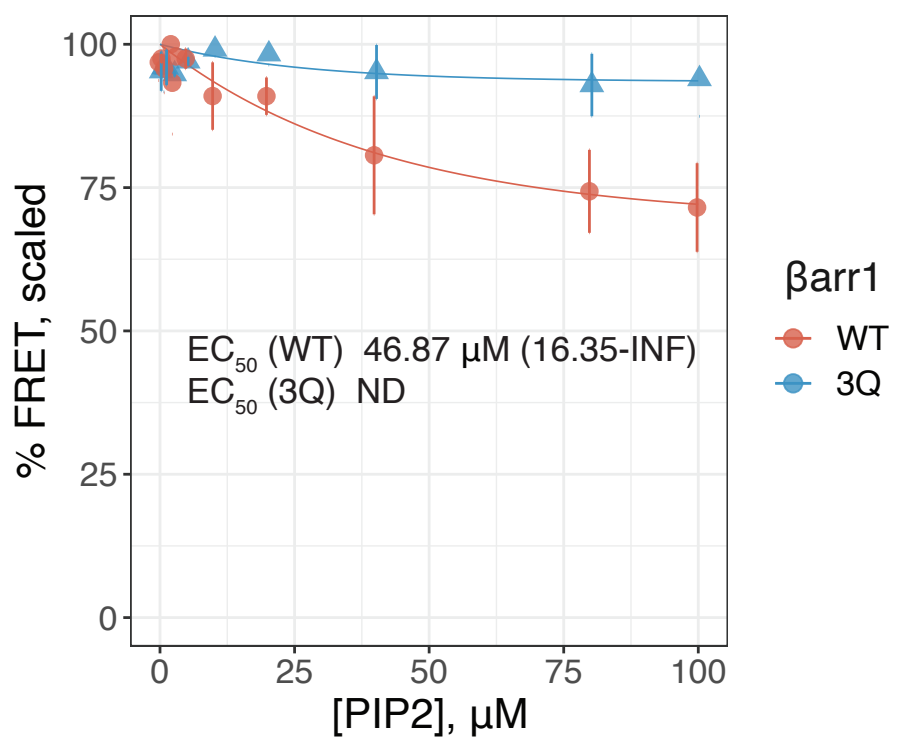

